\title{
Ole e 13 is the unique food allergen in olive: Structure-functional, substrates docking, and molecular allergenicity comparative analysis
}

\author{
J.C. Jimenez-Lopez a,b,*, P. Robles-Bolivar ${ }^{\text {a }}$, F.J. Lopez-Valverde ${ }^{a}$, E. Lima-Cabello ${ }^{a}$, \\ S.O. Kotchoni ${ }^{c, d}$, J.D. Alchéa \\ a Plant Reproductive Biology Laboratory; Department of Biochemistry, Cell E Molecular Biology of Plants; Estación Experimental del Zaidín; Spanish \\ National Research Council (CSIC), Granada 18008, Spain \\ ${ }^{\mathrm{b}}$ The UWA Institute of Agriculture, The University of Western Australia, Crawley, Perth 6009, Australia

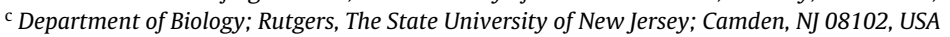 \\ d Center for Computational and Integrative Biology; Rutgers, The State University of New Jersey; Camden, NJ 08102, USA
}

\section{A R T I C L E I N F O}

\section{Article history:}

Received 19 December 2015

Received in revised form 9 February 2016

Accepted 13 March 2016

Available online 17 March 2016

\section{Keywords:}

Catalytic cleft

Electrostatic potential

Food allergy

Homology modeling

IgE-binding and T-cell epitopes

Molecular docking

Olea europaea $\mathrm{L}$

Osmotin

PR5 family

\begin{abstract}
A B S T R A C T
Thaumatin-like proteins (TLPs) are enzymes with important functions in pathogens defense and in the response to biotic and abiotic stresses. Last identified olive allergen (Ole e 13) is a TLP, which may also importantly contribute to food allergy and cross-allergenicity to pollen allergen proteins. The goals of this study are the characterization of the structural-functionality of Ole e 13 with a focus in its catalytic mechanism, and its molecular allergenicity by extensive analysis using different molecular computeraided approaches covering a) functional-regulatory motifs, b) comparative study of linear sequence, 2 -D and 3D structural homology modeling, c) molecular docking with two different $\beta$-D-glucans, d) conservational and evolutionary analysis, e) catalytic mechanism modeling, and f) IgE-binding, B- and T-cell epitopes identification and comparison to other allergenic TLPs.

Sequence comparison, structure-based features, and phylogenetic analysis identified Ole e 13 as a thaumatin-like protein. 3D structural characterization revealed a conserved overall folding among plants TLPs, with mayor differences in the acidic (catalytic) cleft. Molecular docking analysis using two $\beta-(1,3)-$ glucans allowed to identify fundamental residues involved in the endo-1,3- $\beta$-glucanase activity, and defining E84 as one of the conserved residues of the TLPs responsible of the nucleophilic attack to initiate the enzymatic reaction and D107 as proton donor, thus proposing a catalytic mechanism for Ole e 13 . Identification of IgE-binding, B- and T-cell epitopes may help designing strategies to improve diagnosis and immunotherapy to food allergy and cross-allergenic pollen TLPs.
\end{abstract}

(C) 2016 Elsevier Inc. All rights reserved.

\section{Introduction}

Thaumatin-like proteins (TLPs) are structurally quite similar, with conserved domains in several regions of the protein [1]. They are proteins highly synthetized in response to biotic and environmental stresses [2], and in some cases giving sweet taste to foods, particularly fruits [3].

TLPs belong to the pathogenesis-related (PR) protein family 5 (PR5) (one of 17 distinct PR protein families) based in their sequence similarity [4,5], which represent a divergent group of

\footnotetext{
* Corresponding author at: Plant Reproductive Biology Laboratory; Department of Biochemistry, Cell \& Molecular Biology of Plants; Estación Experimental del Zaidín; Spanish National Research Council (CSIC), Granada 18008, Spain.

E-mail addresses: josecarlos.jimenez@eez.csic.es, jcjimenezl75@gmail.com (J.C. Jimenez-Lopez).
}

protein families involved in plant defense functions and different kinds of responses to stresses, i.e. the osmotin (cumulates in response to both biotic and abiotic stresses-antifreeze activity-and exhibits antifungal activities), permeatins (present in cereal seeds), including zeamatin from Zea mays, hordomatin from Hordeum vulgare, and avematin from Avena sativa [6,7]. TLPs are classified into three groups: those produced in response to (i) pathogen infection, (ii) osmotic stress (also called osmotins), and (iii) antifungal proteins present in cereal seeds. At present, the basic mechanism of this antifungal activity is still not fully understood, although it may be related to a membrane-permeabilizing activity of the pathogens [8], and involves the typical structural feature such as an acidic cleft in thaumatin-like proteins [9]. The predicted mechanism throughout which the PR-5 proteins exhibit antifungal activity is probably the disruption of the proper assembly of the fungal cell wall during hypha elongation by binding and degrada- 
tion of nascent $\beta$-(1,3)-D-glucan molecules [10]. The affinity of PR-5 proteins for carbohydrates varies among proteins [11,12], thus, these differences might explain differential antifungal specificities among fungal species [10].

The availability of information concerning sequenced genomes is making possible the identification of the TLP gene superfamily in Oryza sativa, Arabidopsis thaliana, Picea glauca, Pinus monticola, and mosses. However, no TLP gene has been retrieved from the green alga Chlamydomonas reinhardtii [2].

TLPs have been identified in several organisms, being universal in plants including both gymnosperms and angiosperms [2,5], and bryophytes like the moss Physcomitrella patens subsp. Patens. In some species of plants, TLPs are constitutively expressed in flowers and fruits, where they perform defense functions against infection [11]. TLPs are also induced in response to wounding and insect feeding [12]. Some TLPs with antifungal activity act by permeabilizing fungal membranes [13]. Other TLPs appear to function by inhibiting fungal xylanases [14]; potentially as $\alpha$-amylase and trypsin inhibitors [15]; or by binding and hydrolyzing $\beta$-(1,3)-glucans [16] throughout unclear mechanisms of binding and catalytic activity. The hydrolytic activity of complex sugars (i.e. $\beta$-glucan oligomers with different degrees of polymerization) seems to elicit and induce various defense responses of the plants, such as the activation of chitinase activity [18], and other defense responses in tobacco [17]; the specific degradation of cell wall $\beta-(1,3)$-D-glucans [18]; or a contribution to the softening of the pulp or flesh of fruits as grape and cherry [19] in ripening fruits.

On the other hand, pathogenesis-related proteins are reported to be allergens [20], particularly those of the PR-5 family. A limited number of TLPs have been identified from pollens and plant foods as allergens in susceptible atopic individuals [20], due to lack of studies involving molecular allergy. Among these TLPs from pollen, the following could be highlighted: Jun a 3 [21], Cup a 3 [22], and Cry j 3 [23] allergens. Among foods, the following allergenic proteins are included: Lyc e NP24 [24], Pru av 2 [25], Act c 2 [26], Mal d 2 [27], Cap a 1 [28], Vit v TLP [29], and Mus a 4 [30]. Although there is no currently experimental evidence, their wide distribution (ubiquitous location) in plant species, and their allergenic properties, (i.e. cross-reactivity between pollen and fruit as PR-5 allergens, likely due to sharing similar structures), strongly indicate that this family of proteins might be proposed as a panallergen, similarly to profilin, LTP [31].

To date, twelve allergens have been identified and characterized in olive tree pollen. However, Ole e 13 is the only food allergen identified, until now, in the olive fruit. In the current study, we performed an extensive structure-functional, molecular interaction and conservational analysis of Ole e 13 allergen protein in comparison to other allergenic TLPs by using homology modeling and protein-ligand docking methods, defining the catalytic-binding cleft for different substrates. Furthermore, the potential key residues for enzyme activity and substrate specificity were highlighted, and a putative catalytic mechanism was proposed for this protein. We also identified the sequences of the IgE, B- and T-cell epitopes of Ole e 13, and perform a molecular analysis of possible cross-reactivity between food and/or pollen allergens. Structural knowledge of the epitopes responsible for allergy of these proteins is essential to design therapeutic tools (i.e. immunotherapy) to tackle allergy.

\section{Material and methods}

\subsection{Thaumatin-like protein sequences retrieval}

Olive TLP (Ole e 13 allergen) sequence (NCBI accession number EU927297) was used as query to search TLPs against publicly available sequence databases Swiss-Prot/TrEMBL (Uniprot) (http:// www.uniprot.org/), and NCBI (http://www.ncbi.nlm.nih.gov/), by using BLASTX, BLASTN and BLAST (low complexity filter, Blosum62 substitution matrix) (http://blast.ncbi.nlm.nih.gov/Blast. cgi/). Others databases were searched looking for other allergenic TLPs (Allergome, http://www.allergome.org/); SDAP, https:// fermi.utmb.edu/), Allergen online (http://www.allergenonline. $\mathrm{com} /$ ); and FARRP databases (http://farrp.unl.edu/resources/farrpdatabases).

TLPs characteristic patterns, as well as functional (biologically meaningful) motifs were analyzed for each sequence by using the PROSITE database (http://prosite.expasy.org/).

\subsection{Phylogenetic analysis of thaumatin-like proteins}

Amino acid sequences retrieved from 56 TLPs were used to make multiple alignments by using ClustalW tools (http://www.ebi.ac. uk/Tools/clustalw/). These alignments were made using the Gonnet protein weight matrix, multiple alignment gap opening/extension penalties of 10/0.5 and pairwise gap opening/extension penalties of $10 / 0.1$. The outputs were manually checked to optimize the alignment by using Bioedit v7.0.5.3 (www.mbio.ncsu.edu/bioedit/ bioedit.html). Phylogenetic trees were generated by the neighborjoining method (NJ), and the branches were tested with 1000 bootstrap replicates. Trees were visualized by using Treedyn (www. treedyn.org).

\subsection{Template searching for thaumatin-like protein models building}

Protein Data Bank (PDB) was searched for Ole e 13 protein homology. The best homologous templates for Ole e 13 and TLPs from other plant species were selected by BLAST server (http://ncbi. nlm.nih.gov/). The BioInfoBank Metaserver (http://meta.bioinfo. $\mathrm{pl} /$ ) and Swiss-model server for template identification (swissmodel.expasy.org) were also used for templates selection. The best templates (1Z3Q, 1AUN, 1PCV, and 1DU5) were retrieved from PDB database and used for homology modeling.

\subsection{Homology modeling, structural comparisons and evolutionary conservation analysis of thaumatin-like proteins}

TLPs protein retrieved sequences were used to build a protein model by using the closer PDB template structures by SWISSMODEL (swissmodel.expasy.org). An initial structural model was analyzed for recognition of errors in 3D structure by using ProSA (prosa.services.came.sbg.ac.at/prosa.php), and for a first overall quality estimation of the model with QMEAN (swissmodel.expasy. org/qmean/cgi/index.cgi). Final TLP structures were subjected to energy minimization with GROMOS96 force field energy implemented in DeepView/Swiss-PDBViewer v3.7 (spdbv.vitalit.ch) to improve the van der Waals contacts and correct the stereochemistry of the model. The final quality of the models was assessed by QMEAN, the stereology with PROCHECK (www.ebi.ac.uk/thorntonsrv/software/PROCHECK), and ProSA (prosa.services.came.sbg.ac. at/prosa.php) programs, and the protein energy with ANOLEA (protein.bio.puc.cl/cardex/servers/anolea). The Ramachandran plot statistics for the models were calculated to show the number of protein residues in the favored regions.

Superimposition method of the structural $C \alpha$ carbons of the final built models was used for structural similarity comparisons. The different protein structural features were visualized in PyMol software (http://www.pymol.org/). Conservation scores were obtained by ConSurf server (consurf.tau.ac.il). Therefore, identification of ligand-binding domains in the thaumatin-like protein structures was performed using Cofactor software (zhanglab. 
ccmb.med.umich.edu/COFACTOR). The identification of functional analogs of the query proteins was possible by Gene Ontology (GO) terms (The Gene Ontology project) (www.geneontology.org).

\subsection{Thaumatin-like proteins electrostatic potential analysis}

Electrostatic potential was assessed for all the TLPs structures by using APBS (DeLano Scientific LLC) molecular modeling software implemented in PyMol 0.99 (www.pymol.org). AMBER99 assigned the charges and radii to all of the atoms, and was optimized with the Python software package PDB2PQR. Fine grid spaces of $0.35 \AA$ were used to solve the linearized $\mathrm{PB}$ equation in sequential focusing multigrid calculations in a mesh of 130 points per dimension at $310.00 \mathrm{~K}$. The dielectric constants were 2.0 for the protein and 80.00 for water. The output mesh was processed in the scalar OpenDX format to render isocontours and maps onto the surfaces with PyMOL 0.99. Potential values are given in units of $\mathrm{kT}$ per unit charge $(\mathrm{k}$ Boltzmann's constant; T temperature).

\subsection{Olive thaumatin-ligands ( $\beta$-1,3-D-glucans) molecular docking}

Molecular docking process was performed for olive thaumatinlike protein and two different $\beta-(1,3)$-D-glucans: curdlan (a water insoluble $\beta-(1,3)$-D-glucan), and a chain of six residues of $\beta-(1,3)-$ D-Glcp built by using the SWEET2 modeling (GlycoSciences, http:// www.glycosciences.de/modeling/index.php).

Using these sugar molecules and the three-dimensional molecular modeling of olive thaumatin-like protein, docking studies were performed using two approaches. First one involves the use of SwissDock based on EADock DSS [32], whose algorithm consists of i) generation of binding modes either in local and blind docking, ii) estimation of CHARMM force field energies with GRID, iii) evaluation of binding modes with the most favorable energies with FACTS, and clustering, iv) visualization of the most favorable clusters. The best scoring docked models exhibiting the best superposition with ligands and displaying the lowest binding energy are analyzed.

As a second approach, these docking studies were also comparatively performed with FireDock [33], and algorithm for refinement and re-scoring of rigid-body protein-protein/ligands docking methods performed by Patchdock [34].

\subsection{Allergenicity profile assessment of olive Ole e 13 and other TLPS}

Food Allergy Research and Resource Program (FARRP) allergen protein database (www.allergenonline.org) was used for assessing the allergenicity of olive thaumatin-like protein and other species counterparts, according to the FAO/WHO guidelines. Similarly, allergenicity was also predicted using Allermatch web tool (http:// allermatch.org/), Evaller (http://www.slv.se/en-gb/Group1/FoodSafety/e-Testing-of-protein-allergenicity/), and SDAP, the Structural Database of Allergenic proteins (http://fermi.utmb.edu/). Peptides were selectively compared against either allergenic and non-allergenic peptides in the database.

\subsection{IgE-binding epitopes identification for Ole e 13 and other allergenic TLPS}

AlgPred server (http://www.imtech.res.in/raghava/algpred/ submission.html) was used to identify IgE-binding epitopes for olive Ole e 13 and other species counterpart proteins. This is a similarity-based approach, whereby a protein is predicted to be an allergen if it has a region/peptide identical to a known IgE epitopes.
Sensibility cut-off was established as 80,60 and 50 for epitopes having residues $<10$, between 10 and 15 and $>15$, respectively.

\subsection{T-cell epitopes identification for Ole e 13 and other allergenic TLPS}

Stabilization matrix alignment methods were used to identify peptide of Major Histocompatibility Complex (MHC) binding: TEPITOPE (www.bioinformation.net/ted), Propred (www. imtech.res.in/raghava/propred) (based in quantitative matrices), NetMHCII (www.cbs.dtu.dk), Multipred (antigen.i2r.a-star.edu.sg/ multipred), CTLpred (www.imtech.res.in/raghava/ctlpred) (artificial neural network approach), and RANKPEP (bio.dfci.harvard. edu/Tools/rankpep.html) (Position-specific scoring matrix), which employ binding status scoring qualitative prediction methods.

\subsection{Linear B-cell epitope identification for Ole e 13 and other allergenic TLPS}

Epitope residue scores were obtained using: ABCpred (www. imtech.res.in/raghava), BepiPred of the IEDB Database use a combination of a hidden Markov model and a propensity scale method (http://tools.immuneepitope.org/tools/bcell/iedb_input), BCpreds (http://ailab.cs.iastate.edu/bcpreds/), and Bcepred (www.imtech. res.in/raghava).

A fixed length epitope prediction of 15 -residues and $80 \%$ specificity was used for scoring potential B-cell epitopes.

The IgE-binding, B- and T-cell epitopes identified by computational tools were mapped on the three dimensional model of Ole e 13 and other allergenic TLPs to determine their position and the secondary structure elements involved.

\section{Results and discussion}

\subsection{Ole e 13 sequence analysis}

Ole e 13 (Uniprot accession number E3SU11) belongs to the thaumatin-like proteins family (CATH Superfamily 2.60.110.10; InterProt number IPR001938, Pfam number PF00314).

Search for characteristic motifs/patterns (http://prosite.expasy. org), and based on PROSITE database of protein domains, families and functional sites, indicated that TLPs are characterized by having a thaumatin family signature (PS00316): G-X-[GF]-X-C-X-T-[GA]-D$C-x(1,2)-[G Q]-x(2,3)-C[35,36]$. In this regard, Ole e 13 exhibits one defined domain integrated by the pattern GRGQCETGDCNGLLEC (position 65-81) (Fig. 1).

The alignment of 15 representative plant TLP sequences shows the diversity of plant TLPs (Fig. 1). Sequences identity comparison has shown a wide range (30.7-92.8\%) of identity among them. Particularly, low identity was found when we compared olive to Arabidopsis (38.7\%), apple (34.9\%), rice (37.3\%), banana (53.8\%), maize (59.4\%) or tomato (59.2\%), but olive showed higher identity with TLPs from kiwi (67.3\%), tobacco PR5 (63.3\%), or bell pepper (62.1\%). Seven are the domains that exhibit relatively high conservation among these TLPs, located in the domain I and III of the TLPS (Fig. 1). Furthermore, amino acid sequences involved in the formation of the bottom of the acidic cleft and those at each side of the thaumatin characteristic motif are well conserved (Fig. 1).

The 16 cysteine residues in the positions $10,59,69,74,76$, $130,135,143,160,164,173,174,184,197,214$ and 226 are well conserved, except for $O$. sativa TLP, which lacked of the C10 in its structure (Fig. 1). The REDDD motif is fully conserved in 14 of the 15 representative sequences, with the exception of thaumatin (Thaumatococcus daniellii). These amino acids are located in a central cleft region. Overall, this acidic cleft is important relevant for an anti- 


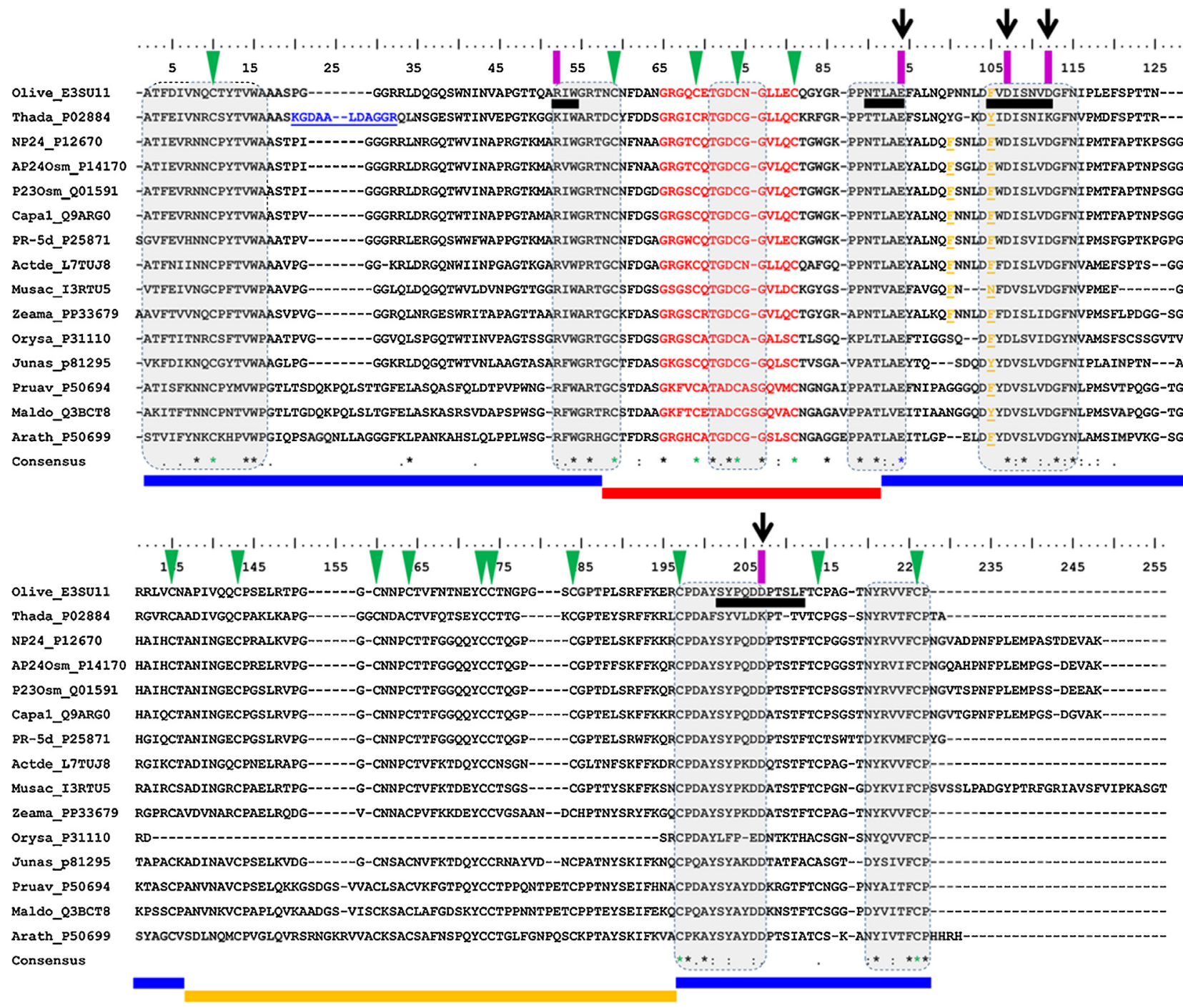

Fig. 1. Sequences analysis of thaumatin-like proteins from different plant species. Sequence comparison of different plant TLPs (Olea europaea E3SU11; Thaumatococcus daniellii P02884; Solanum Lycopersicum P12670; Nicotiana tabacum P14170; Solanum lycopersicum Q01591; Capsicum annuum Q9ARG0; Nicotiana tabacum P25871; Actinidia deliciosa L7TUJ8; Musa acuminata I3RTU5; Zea mays PP33679; Oryza sativa P31110; Juniperus ashei P81295; Prunus avium P50694; Malus domestica Q3BCT8; and Arabidopsis thaliana P50699) was performed by multiple alignment. Conserved residues were highlighted with asterisks, while the highly conserved cysteine residues among plant species are highlighted with green arrows and asterisks. Residues implicated in putative catalytic activity were pointed out with pink rectangles and black arrows. Residues comprising TLPs domain I, II and III are highlighted by blue, yellow, and red rectangles, respectively. Highly conserved areas are highlighted by dashed framed grey rectangles. Residues involved in the acidic cleft side regions are highlighted by black rectangles. Amin acids sequence integrating a characteristic loop in thaumatin $\left({ }^{19}\right.$ KGDAALDAGGR $\left.{ }^{29}\right)$ responsible for its sweet taste is highlighted in blue color. (For interpretation of the references to colour in this figure legend, the reader is referred to the web version of this article.)

fungal activity [37,38], as well as for a $\beta$-(1,3)-glucanase activity [39].

Searching for fundamental amino acids in the alignment (Fig. 1) responsible of other thaumatin-like proteins features, we found that olive and the other TLPs are missing five lysine residues (K78, K97, K106, K137 and K187) that importantly contribute to sweetness in thaumatin I [40]. It has been pointed out that these lysine residues, spread over a broad surface region around the acidic cleft, do not exist in non-sweet TLPs. Furthermore, the positive charges and positions of these lysine residues play an important role in the interaction of thaumatin with human G-protein-coupled receptors (GPCRs). It seems that lysine charge rather than the structure of the side chain is more important for sweetness of thaumatin II [41]. In addition, thaumatin ( $T$. daniellii) exhibited a short sequence of amino acids ( ${ }^{19}$ KGDAALDAGGR ${ }^{29}$ ) integrating a loop. This additional feature may be also responsible for its sweet taste [42]. Indeed, this thaumatin loop sequence is similar to that of peptide sweeteners ( L-Asp-D-Ala-L-Ala-methyl ester and L-Asp-DAla-Gly-methyl ester), which are postulated as one of the important sweet-taste determinants [42] and located in the same side of the cleft region. This 2-D structural element is much shorter in olive Ole e 13 and the other TLPs due to a deletion of six residues (Fig. 1).

Finally, a H22 residue conserved in TLPs is able to form a complex with xylanases, resulting in xylanase-inhibitor capacity [43]. None of the sequences of the alignment of Fig. 1 conserve this residue, and they are thus unable to display this inhibitory capacity. Positive charge of this residue $\mathrm{H} 22$ might be of high relevance for thaumatin-like xylanase interaction and activity [44].

\subsection{Phylogenetic analysis of plant thaumatin-like proteins}

In order to analyze the relationships between TLPs of different plant species, including those from allergenic pollen, we performed 


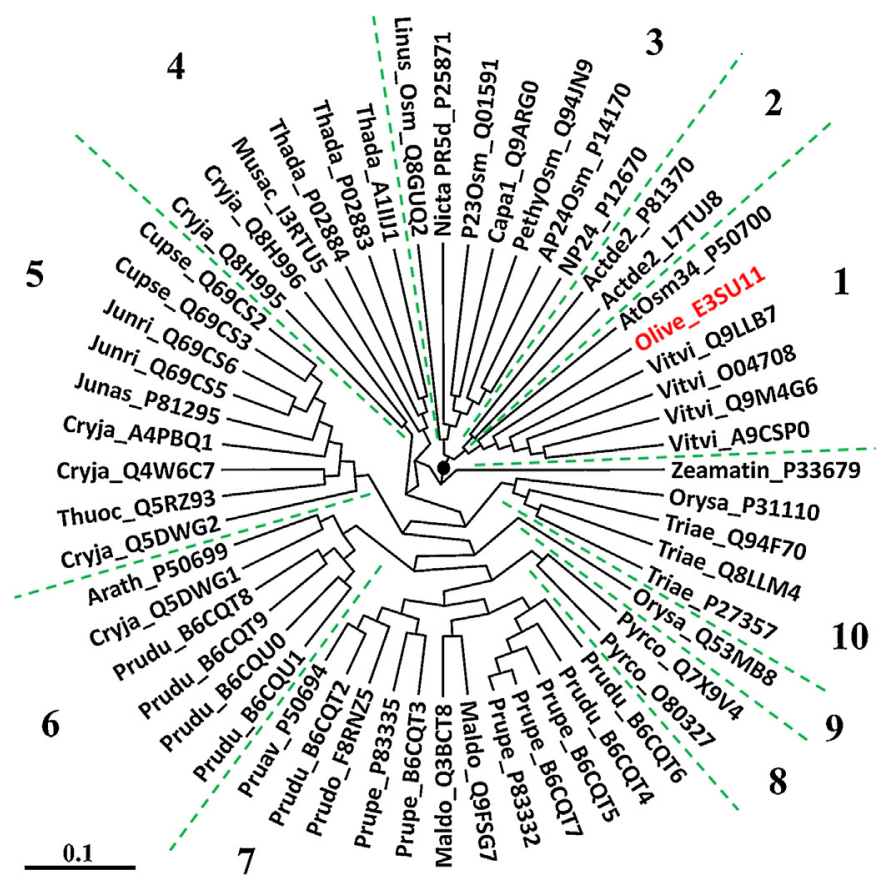

Fig. 2. Phylogenetic analysis of plant thaumatin-like proteins. Neighbor-joining ( $\mathrm{NJ})$ method was used to perform a phylogenetic analysis of 56 TLPs from 24 species such as Actinidia deliciosa (L7TUJ8; P81370), Arabidopsis thaliana (P50699; P50700), Capsicum annuum (Q9ARG0), Cryptomeria japonica (A4PBQ1; Q4W6C7; Q5DWG1; Q5DWG2; Q8H995; Q8H996), Cupressus sempervirens (Q69CS2; Q69CS3), Juniperus ashei (P81295), Juniperus rigida (69CS6; Q69CS5), Linum usitatissimum (Q8GUQ2), Malus domestica (Q3BCT8; Q9F3G7), Musa acuminata (I3RTU5), Nicotiana tabacum (P14170; P25871), Olea europaea (E3SU11), Oryza sativa (P31110; Q53MB8), Petunia hybrida (Q94JN9), Prunus avium (P50694), Prunus domestica (F8RNZ5), Prunus dulcis (B6CQT2; B6CQT4; B6CQT6; B6CQT8; B6CQT9; B6CQU0; B6CQU1), Prunus persica (B6CQT3; B6CQT5; B6CQT7; P83332; P83335), Pyrus pyrifolia (O80327; Q7 × 9V4), Solanum Lycopersicum (Q01591; P12670), Thaumatococcus daniellii (A1IIJ1; P02883; P02884), Thuja occidentalis (Q5RZ93), Triticum aestivum (P27357, Q8LLM4; Q94F70), Vitis hybrid (A9CSP0; 004708; Q9M4G6; Q9LLB7), Zea mays (P33679). Ten defined groups were identified and highlighted by different numbers and green arrows. (For interpretation of the references to colour in this figure legend, the reader is referred to the web version of this article.)

a phylogenetic analysis aiming to gain insights into different functional groups (Fig. 2). 56 sequences of a wide representation of TLPs were aligned and the resulting groups/clusters analyzed. 10 groups were established. The one including olive Ole e 13 protein sequence was defined as group 1. This group was also integrated by TLP sequences from Vitis vinifera, in addition to a sequence from Arabidopsis osmotin 34. The largest group (number 7) was integrated by Prunus genus sequences and two TLPs from Malus domestica. The second largest group (number 5) was mostly constituted by pollen TLP sequences, i.e. from cypress, mountain cedar and Japanese cedar. Other TLP allergenic sequences, such as those from tobacco, chili pepper and petunia were clustered in the group 3 , which was mostly integrated by osmotin functional proteins, while the sequence from kiwi, pear, and rice were independently clustered. This analysis shows that Ole e 13 is closely related to osmotin proteins with antifungal and $1,3-\beta$-glucanase activity.

\subsection{Identification of structural templates for olive and other TLPS}

Ole e 13 structure built and analyzed in the current study is important because it provides for the first time detailed information about the 3D-structure and functionality of an oleaginous plantspecific TLP and, in addition, contributes to a further understanding of the structural features for its putative $1,3-\beta$-glucanase activity and cross-allergenicity between fruit and pollen TLPs. The identification of the protein with the best tertiary structural similarity to Ole e 13 and TLPs from other species was performed. The best threading templates for Ole e 13 were $1 \mathrm{Z3Q}$ (Mus a 4, M. acuminata), 1PCV (Nic t Osmotin, N. tabacum), 1AUN (PR-5d, N. tabacum), and 1DU5 (Zeamatin, Z. mays), whose crystallographic structures (PDBs) were retrieved from Protein Data Bank (PDB). These templates showed highest sequence identities to Ole e 13 of $61.6,58.4$, 57 , and $53.4 \%$, respectively.

After template structures identification, the suitability of these selected models was evaluated by BioInfoBank Metaserver, with calculated 3D Jury score (J-score) of 97.15 (1Z3Q), 91.38 (1PCV), 89.32 (1AUN), and 85.30 (1DU5) for homology modeling of Ole e 13 structure building, respectively. Swiss-model server was also used to perform the assessment of these templates, returning high score of $334,311,294$, and 276 , respectively, and very low E-values of $2 e^{-93}, 5 e^{-87}, 6 e^{-81}$ and $2 e^{-77}$, respectively for the templates. Comparable parameters were found for the assessment of other TLPs models built in the present study.

\subsection{Structure analysis of Ole e 13}

A common feature for both, animal and plant TLPs, is that they contain an $\mathrm{N}$-terminal signal peptide targeting mature proteins into the secretory pathway (http://www.uniprot.org). High number of TLPs belong to the large type of TLPs with molecular masses ranging from 21 to $27 \mathrm{kDa}$, and contain 16 conserved cysteine residues (Fig. 1), in which Ole e 13 is included. On the other hand, small type of TLPs (mostly present in cereals) are around 17-18 kDa. S-type TLPs have only nine to ten cysteine residues at conserved positions as it happens with rice TLP (Fig. 1).

In the current study, we have built the best model for Ole e 13 and other plant TLPs, based in homology modeling. The structural assessment to check the accuracy of the Ole e 13 model was made through a comparative analysis to the templates (crystallographic structures of $1 \mathrm{z} 3 \mathrm{q}, 1 \mathrm{pcv}, 1$ aun, and 1du5) used to build this model, and using stereo-chemical and energy minimization parameters showing the following data:

The analysis of the four best templates showed values of 0.804 , $0.814,0.903$, and 0.884 for the Qmean parameter (linear combination of six terms, including stereology and energy, to estimate the model reliability ranging between 0 and 1), respectively, and 0.795 for Ole e 13 model. Another paramenter to chek the overall quality of the structures, ProSA, showed a z-score of -5.95 for Ole e 13 model, and $-6.31,-5.62,-5.37$, and -6.00 , respectively for the individual four crystallographic structural templates. Both, Qmean and ProSA parameters show values quite similar for the Ole e 13 model compared to the crystallographic structures, which mean that Ole e 13 protein model built is accurate and close to its templates in structure quality.

Therefore, we also check the stereochemistry of the model through Procheck analysis, showing that $86.8,85.0,87.4$, and $84.9 \%$ of the structural residues were located in favorable regions in the respective templates; $12.6,15.0,12.6$, and $15.1 \%$ in allowed regions, $0.6,0,0,0 \%$ in generally allowed regions; and $0 \%$ for the four templates in disallowed regions, when analyzed the Ramachandran plot. These values for Ole e 13 model were 87.1, 12.3, 0.6, and $0 \%$ respectively, finding even more residues located in favorable regions, less residues in allowed regions, and a similar situation in generally allowed and non-favorable regions.

Taking in consideration all these parameters, and the comparison between Ole e 13 and its templates, we can affirm that Ole e 13 protein model build from its crystallographic template is accurate and reliable to be use in further structure-functional analyses. A similar assessment was made for the other TLPs structures built, and similar results were found when compared to their templates.

The overall structure of Ole e 13 (Fig. 3A) exhibits the characteristic thaumatin-like fold, including three domains (I, II, and III), 


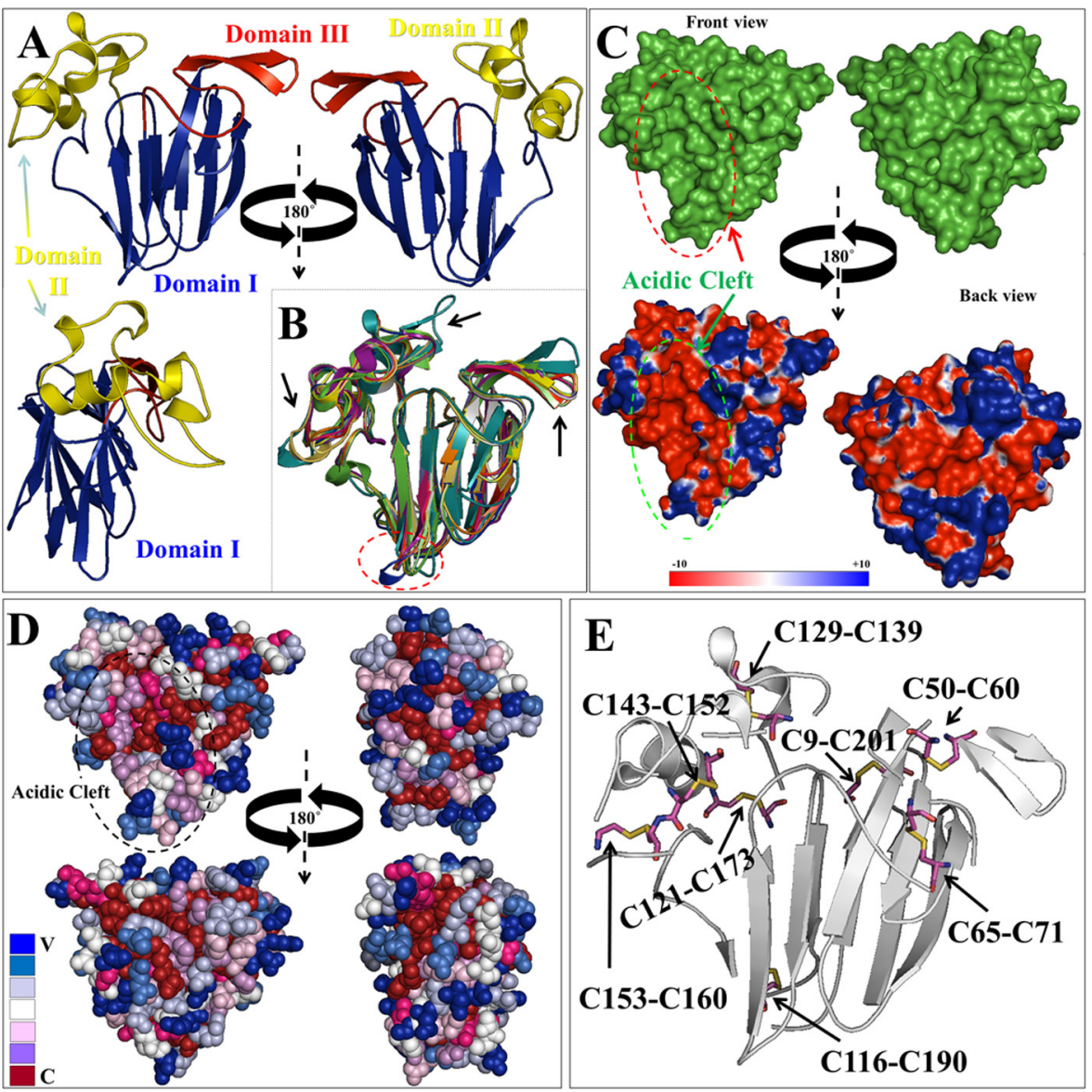

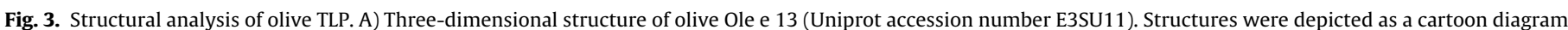

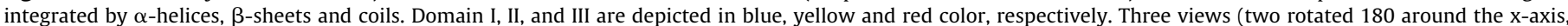

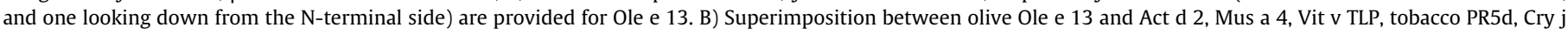

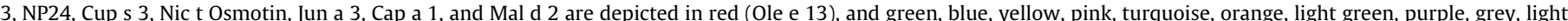

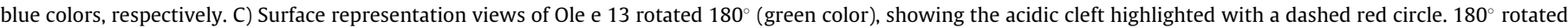

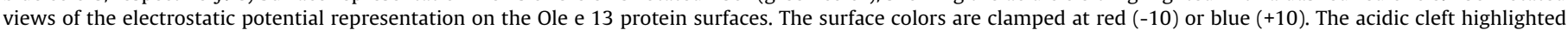

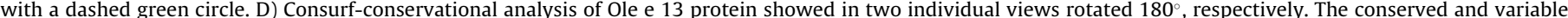

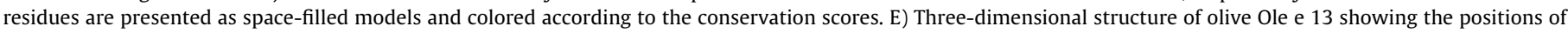
eight disulphide bridges. (For interpretation of the references to colour in this figure legend, the reader is referred to the web version of this article.)

where an acidic cleft is located between the central domains I and II (Fig. 3) [39]. Domain I (A1-T48, L81-N122, C173-P202) forms the central core of the molecule, integrated by $11 \beta$-strands. Domain I is a lectin-like $\beta$-barrel that forms the compact core of a TLP molecule. To one side of domain I and domain II, a large disulphide-rich $\alpha$-helical region including 6 cysteine residues, comprised by A123R172, and an extended $\alpha$-helix 4 associated with three shorter $\alpha$-helices $\alpha 1, \alpha 2, \alpha 3$ made up of several loops and partial $\alpha$-helices. These domains delineate a central acidic cleft which contains predominantly hydrophilic residues. On the other side is domain III (N49-T80), which consists of a small loop (hairpin segment) and two antiparallel $\beta$-strands linked to an extended loop (Fig. 3A). Throughout the comparison of different plants TLPs by superimposition (Fig. 3B), we can delimit that the overall 3D structure is quite similar, and Ole e 13 well superimposes with other known TLPs (Fig. 3B). However, differences are shown in the loop integrating the lower part of the acidic cleft, and the size of loops in domains II and III. Six antiparallel $\beta$-strands $(\beta 2, \beta 3, \beta 5, \beta 6, \beta 7$ and $\beta 10)$ form the front area of the domain I and the other five $(\beta 1, \beta 4, \beta 8, \beta 9$ and $\beta 11$ ) form the back area of this domain, which together constitute the $\beta$-sandwich. Comparison between Ole e 13 and various TLP structures gave superimposition values of $0.271 \AA$, $0.279 \AA, 0.333 \AA$, $0.367 \AA, 0.4 \AA, 0.401 \AA, 0.403 \AA, 0.409 \AA, 0.413 \AA$, and $1.197 \AA$ for Act d 2 and Mus a 4, Vit v TLP, tobacco PR5d, Cry j 3, Sola 1 TLP/NP24, Cup s 3, and Nic t Osmotin, Jun a 3, Cap a 1, and Mal d 2, respectively.

Surface of Ole e 13 (rotated $180^{\circ}$ ) is depicted in Fig. 3C. The morphology of the cavity that accommodates the substrate (i.e. 1,3$\beta$-D-glycan) of the TLPs is integrated by a solvent-accessible cleft in between domain I and II of the protein, formed by the external loops and front $\beta$-strands of domain I (Fig. 3C). The central part of this cleft is lined by several polar (negative) residues, among them the REDDD motif $\left(\mathrm{R}^{52}-\mathrm{E}^{94}-\mathrm{D}^{107}-\mathrm{D}^{112}-\mathrm{D}^{207}\right)$ involved in the catalytic mechanism of the Ole e 13.

Surface electrostatic potential analysis, reveals a dominance of negative charges over the surface of the protein, substantially patent in the acidic cleft in the domain I (Fig. 3C). Several prominent charged residues exhibiting large positive values, blue regions, dominate the domain III, and the lower part of the acidic cleft extending to the back side of the domain I. By assigning a value of +1 to basic residues $(R, K)$ and -1 to the acidic residues $(D, E)$, net charge of protein was calculated to be -7 for Ole e 13 .

Consurf conservational analysis has shown that TLPs are not well-conserved protein (Fig. 3D) when comparison is made of animal, plant, bacterial and fungi species. We found different residues spread in the surface of the protein, particularly in areas of the plant TLPs sequences out of the not conserved domains (purple color) (Fig. 1). The only region of the protein which can be considered as 
highly conserved is the one integrating the core of the acidic cleft where key catalytic residues are located (Fig. 3D). Several others amino acids with a major role in the maintenance of the protein structure are also conserved.

Among the three domains that constitute Ole e 13, a total of 16 cysteine residues were found, which exhibited a conserved spatial distribution throughout the TLPs [38]. These residues can take part of up to 8 disulphide bridges, highly conserved in TLPs $[15,30,37,38]$. They contribute constitute an important feature of the protein participating in the correct folding to TLPs, and giving high stability under thermal and $\mathrm{pH}$ conditions [44], as well as resistance to protease degradation [45]. Each domain is stabilized by at least one disulphide bridge (Fig. 3E). In addition, domains I and II, and I and III are linked by at least one disulphide bridge. The 2-D structural elements, particularly the loops linked by two cysteine residues exhibited similar topology as established by threading homology modeling for other plants and animals TLPs [46].

\subsection{Comparative analysis of the electrostatic potential concerning the Ole e 13 substrate binding cleft}

Strong evidence suggested that antifungal properties of some TLP are closely associated to the prominent acidic (electronegative) cleft located between domains I and II. This cleft is a distinctive feature of the TLPs [37], as identified in the crystal structure of zeamatin [6], PR-5d [7] and osmotin [38] from tobacco, and predicted by structure modeling in apple [45], cherry [25], and now in Ole e 13 [current study].

Electrostatic modeling of Ole e 13 reveals a highly acidic cleft, an extended structure located between domains I and II, where most part of it is in the front view of the domain I (Fig. 4), and dominated mainly by hydrophilic residues, and surrounded by aromatic residues Y74, P89, F94, F103, Y177 and Y179 (Figs. 1 and 3). It is interesting, however, that the back surface of Ole e 13 (as it happens with thaumatin), is dominated by basic nature (positive residues) (Fig. 3B).

The Fig. 4 shows the cleft, which is electronegative in all TLPs. This acidic character depends on $\mathrm{pH}$, at $\mathrm{pH} 5$, Mal d 2 and Pru av 2 display a less widespread electronegativity cleft due to the location of the side-chains of some acidic residues ( $D$ and $E$ ) protruding from the surface of the cleft. This cleft is particularly rich in acidic residues in Ole e 13 (E84, D107, D112, D207) and accordingly it corresponds to a region with strong electronegative (acidic) character (Figs. 3 and 4). The main residues contributing to the acidic environment of the cleft are R52, R56, D73, E84, D107, D112, D206 and D207. Most of these residues are also found in TLPs such as PR5d $[7,13]$.

This electronegative property of this cleft might represent a predefined requisite, as it happens with PR-5 proteins, where it enables them to interact with positively charged membrane proteins, i.e. ions or water channels [47] and become one type of antifungal defense mechanism. At the same time, this is a pre-requisite of some TLPs exhibiting endo-1,3- $\beta$-glucanase activity. Glucanase activity of TLPs with strong electronegativity could range between different levels of activity. Thus, Mus a 4 displays a moderate level of antifungal activity (probably without biological relevance) [30]. NP24 has low endoglucanase activity [24] to hydrolyze water soluble $\beta$-(1,3)-D-glucans after extended incubation. Finally, Mal d 2 (an allergenic TLP from apple) shows extremely weak activity under in vitro antifungal assays against Fusarium oxysporun and Penicillium expansum [27], in comparison whit the genuine antifungal TLP (like osmotin). This may suggest that other structural features, could be of relevance and maybe more important for antifungal activity, since thaumatin (which has a predominantly basic (electropositive) cleft region), has been reported to have weak antifungal activity [48].
Furthermore, this acidic cleft may have other relevant structural requirements, as well as mayor acidic, neutral or basic nature to accommodate and bind different ligands/receptors, i.e. olive Ole e 13 , tobacco, mountain cedar, tomato are examples of large acidic clefts, while $T$. daniellii or grape have catalytic clefts with lower acidic properties (Fig. 4), and the replacement of some of these amino acids by other of neutral or acidic nature can modify the electronegativity of the top of the cleft.

In all plant proteins from the pathogen-related 5 family, with known antifungal activity this cleft is acidic because of the presence of a conserved motif of five amino acids (REDDD or arginine, glutamic acid, and three aspartic acid residues) (Fig. 1). This acidic cleft might be relevant to their specific receptor binding for an antifungal activity $[15,37,38]$. An example of basic cleft is thaumatin with a differential 2-D element (a loop within the domain I), which is absent in the antifungal plant PR5 proteins, thus explaining their differences in function [38].

It has been probed the link between the acidic characteristics of the cleft and the antifungal activity in TLPs [12,30] as result of adaptive evolution to environmental stresses that can alter the antifungal function of TLPs through alternation of the electrostatic potential of the acidic cleft and/or the molecular surface. In this regard, a K49 present in thaumatin and NP24 proteins is substituted in Ole e 13 by an $\mathrm{R}$ with positive charge that is important for the basic character of other TLPs as thaumatin. Furthermore, aromatic residues as F90, F95 and K179 (P90, F95 and K175 in Ole e 13) are surrounding its acidic cleft, and three conservative sites (R43, R47, and R157) are located in the cleft or on the surface of the cleft. In addition, a big differential feature in Ole e 13 and other TLPs that markedly contributes to the acidic cleft conservation is the absence of $\mathrm{K}$ residues surrounding the cleft region in thaumatin, which are implicated in the sweet property [40]. These Lys residues are substituted in Ole e 13 by Q73, L93, D185 and E136.

\subsection{Structural features responsible for the 1,3- $\beta$-glucanase and putative antifungal activity in Ole e 13}

A mayor difference among thaumatin-like proteins relies in the distribution of charges around their surface (Fig. 4). On the opposite, the electronegative character of the catalytic cleft of many TLPs is a specific feature of this PR5 family, making it a crucial contribution towards their $\beta$-(1,3)-glucan binding and 1,3- $\beta$-glucanase activities [7,13]. Many antifungal TLPs can either bind or cleave simple or complex polymeric glucans [10-12,17,49], with optimal enzymatic activity developed at $\mathrm{pH}=5$. Furthermore, there might be a correlation between antifungal and 1,3- $\beta$-glucanase activities of TLPs, due to their ability to bind and degrade $\beta-(1,3)$-D-glucans, which are one of the major constituents of fungal cell walls.

Various amino acids such as E84, D107, D112 and D207 are located in the central cleft region of Ole e 13 (Fig. 5A), likely contributing to the arrangement of the catalytic E-D pair required for the $\beta$-(1,3)-glucanase activity. Two acidic amino acid residues located in the clef (glutamic acid and aspartic acid), are suggested to play a role in the nucleophilic attack, and as proton donors in the cleavage of $\beta-(1,3)$-D-glucan or polyglucan (glycosidic bond) [17]. The distance between different E-D pairs are $3.28 \AA, 7.58 \AA$, and 8.91 Å for E84-D107, E84-D112, and E84-D207, respectively. These E-D pair with proper orientation and the correct distance would be able to act as the catalytically active center of TLPs $[17,39]$. This endo-(1,3)- $\beta$-glucanase activity has been also determined for other TLPs as cherry and tomato [12,17], where the presence of a comparable catalytic active center (E71-E150 pair and E84-D102 pair) has been described [17,39]. In addition, two acidic amino acid pairs (E92-D110 and D71-D150) were also described as potential catalytic center for glucanase activity in Populus TLPs (Fig. 3). 


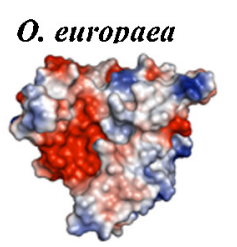

Ole e 13

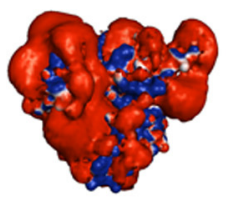

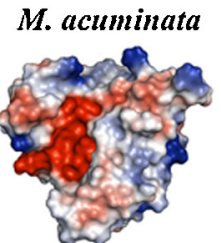

Mus a 4

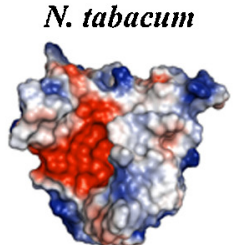

Nic $t$ Osmotin

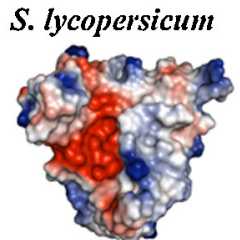

Sola 1 TLP/NP24

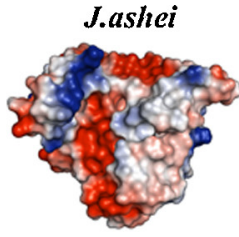

Jun a 3
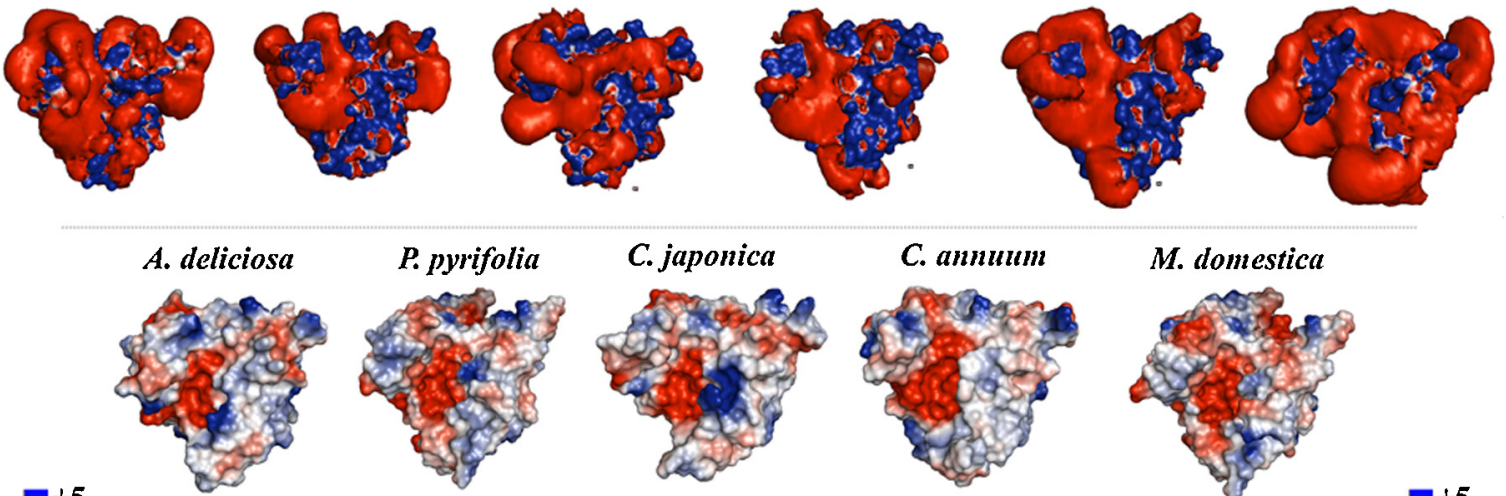

C. japonica

$$
\text { C. annutum }
$$

M. domestica
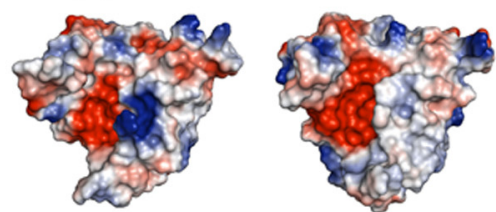

Act d 2
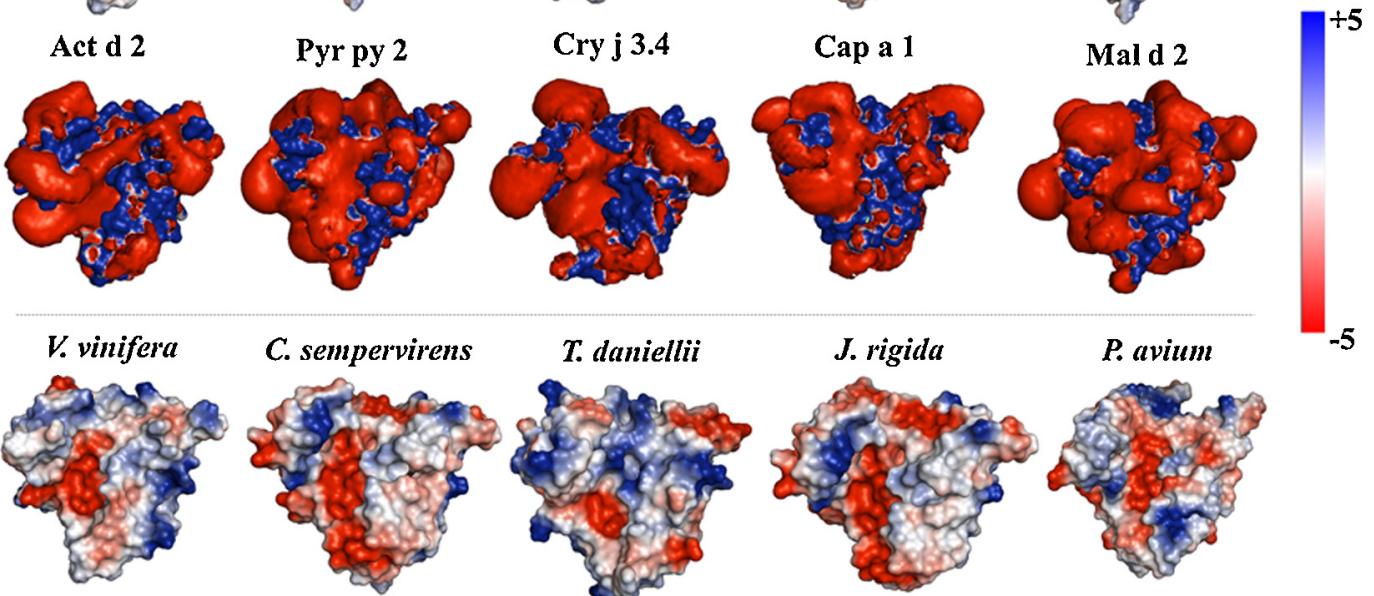

T. daniellii

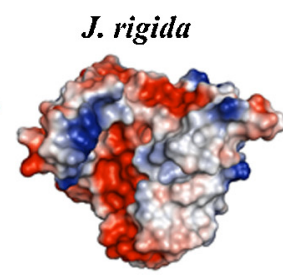

P. avium
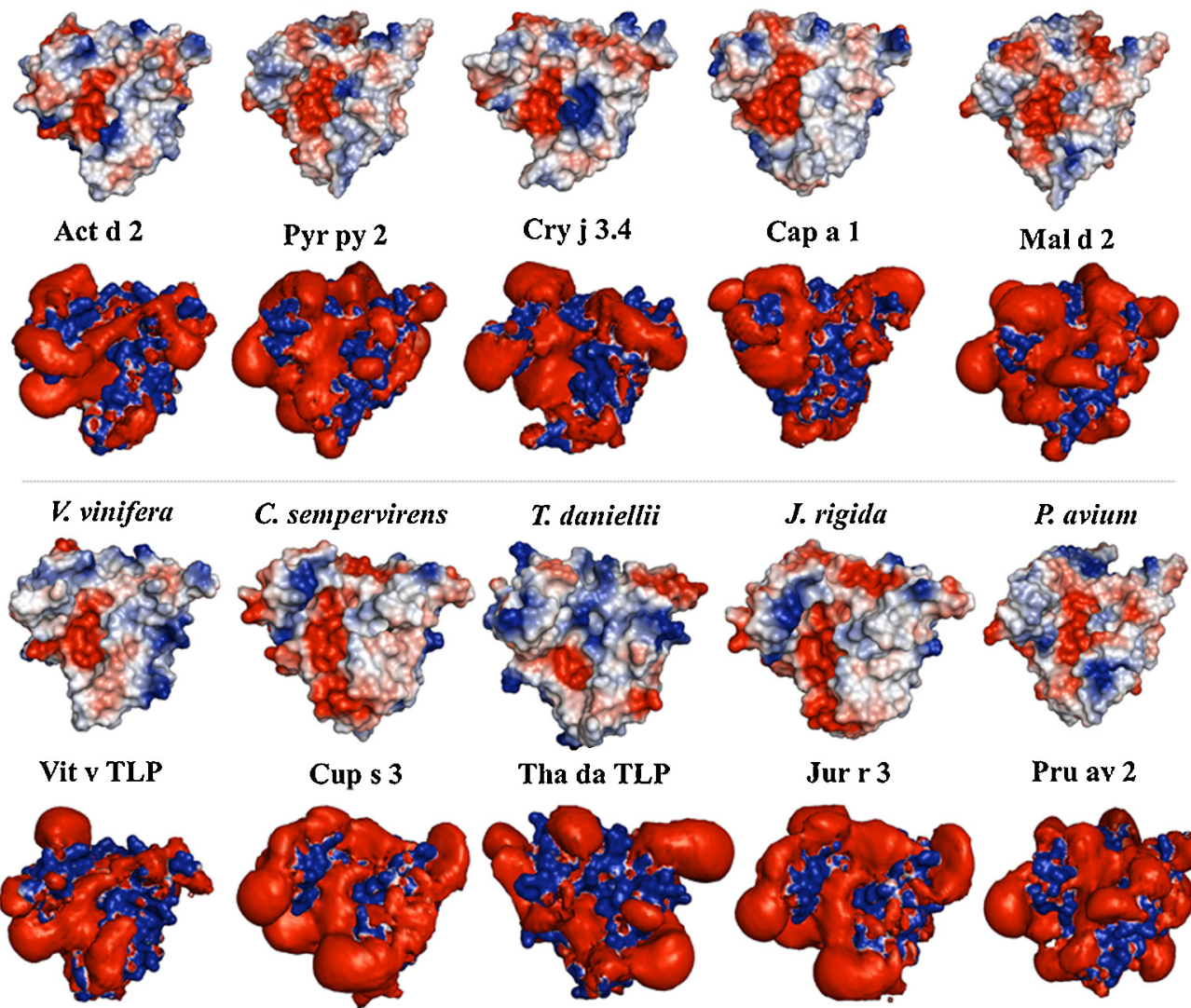

Tha da TLP

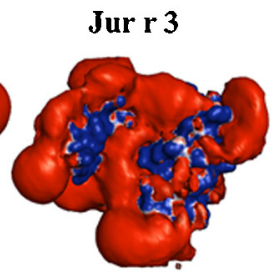

Pru av 2
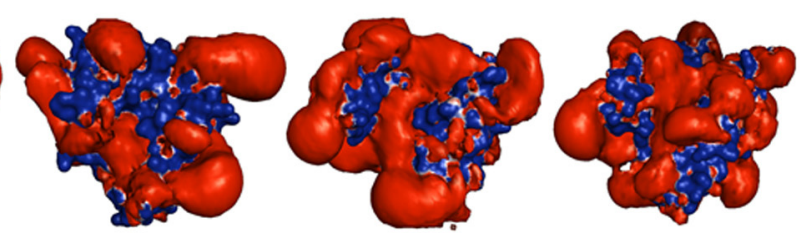

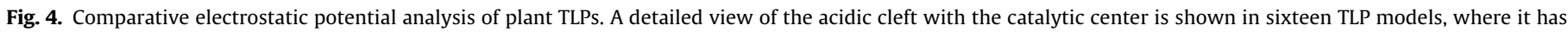
been depicted the electrostatic potential (isocontour value of $\pm 5 \mathrm{kT} / \mathrm{e}$ ) in the surface.

A comparative analysis of the catalytic pair distance was performed between Ole e 13 and these know glucanases. E92-D110 pair distances between $\mathrm{O} \varepsilon$ of $\mathrm{E}$ and $\mathrm{O} \delta$ of $\mathrm{D}$ vary from 6.90 to $7.58 \AA ̊$ in Populus. However, it has been established that the average distance between the carboxylic oxygen atoms of the two catalytic residues for glucan hydrolysis in glycosidases is $\sim 5 \AA$ [50]. Additionally, it has been also demonstrated in other species that the distance of the retaining and inverting mechanism for glycan hydrolysis by TLPs ranges between $5.5 \AA$ and $6.5-9.5 \AA$ (i.e. it has value of $7.93 \AA$ in cherry TLP, $7.07 \AA$ in $2 A H N, 5.90 \AA$ in $1 Z 3 Q$, and $7.07 \AA$ in $2 \mathrm{IOW}$ ) [17]. In summary, distances longer than $5 \AA$ between catalytic E-D pair may result in lower level of its activity for hydrolytic cleavage of glycan polymers by a lack of proper environment. Similarly, too short distance separation (less than $3 \AA$ ) makes E-D pair unlikely to act as catalytically active residues [39].
On the basis of the above information, the most probable

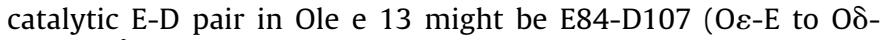
$\mathrm{D}=3.28 \AA$ ). . However, other possible catalytic E-D pairs as E84-D112 (7.58 $)$, and E84-D207 (8.91 $)$ ), cannot be ruled out. They may be involved in the cleavage of the (1,3)- $\beta$-D-glycan chain with lower level of glucanase activity in different types of glucans, depending of their accommodation (orientation and distance to E84, and its $\mathrm{D}$ catalytic partners), and in a location of lowest binding energy into the cleft. In this regard, it has been proposed a catalytic mechanism for Ole e 13 as endo-(1,3)- $\beta$-glucanase (Fig. 5B) where E84 would be the residue performing the nucleophilic attack over the 1,3 - $\beta$-glycoside bond, and D107 being the proton donor. In a proposed mechanism, for each step, there is an oxocarbenium ion-like transition state. Reaction occurs with acid/base and nucleophilic assistance, typically provided by glutamate or aspartate, located about $5 \AA$ apart (Fig. $5 A$ ). In a first step, one residue (E) plays the 

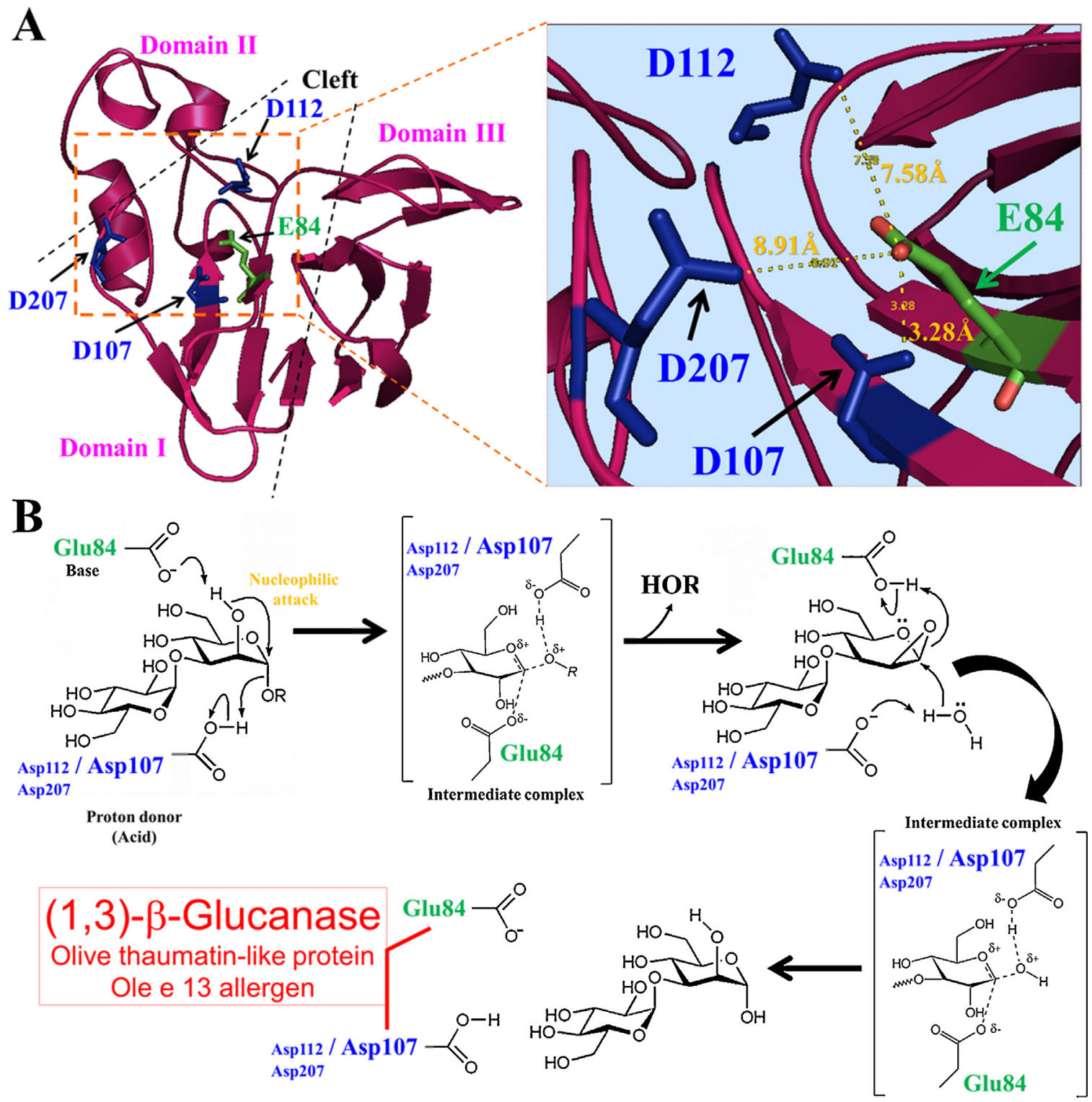

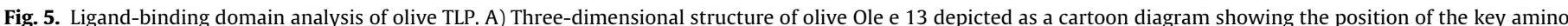

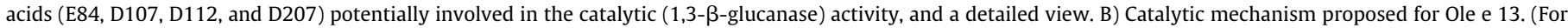
interpretation of the references to colour in the figure legend, the reader is referred to the web version of this article.)

role of a nucleophile, attacking the anomeric center to displace the $\alpha$-glycan and to form a glycosyl enzyme intermediate (Fig. 5B). At the same time the other residue functions as an acid catalyst and protonates (D) the glycosidic oxygen as the bond cleaves. In a second step, the glycosyl enzyme is hydrolyzed by water, with the other residue now acting as a base catalyst (D) deprotonating the water molecule as it attacks (Fig. 5B).

Docking simulations were used to reveal the catalytic mechanism, the glucan binding properties and glucanase potential of the olive Ole e 13. We used two types of glycan molecules, i) a chain consisting of five $\beta-(1,3)-D-g l u c a n$ units, and ii) curdlan, a water insoluble $\beta-1,3-D-$ glucan.

In the first case of docked complex (Fig. 6A), the core region of the $(1,3)-\beta$-D-glucan passed through the acidic inter-domain cleft of Ole e 13, and positioned very close to two acidic residues (E84 and D107), not in the middle of both residues, and a bit further from the other D partners. This docking position revealed that those glycan chains were having the lowest binding energies compared to a lying-down at different locations on the surface of the Ole e 13, which is based on the hydrophobic interactions of the glucose units with apolar and aromatic residues protruding from the surface of the cleft. The calculated distance between the carboxylic oxygen atoms of the catalytic pair is $3.28 \AA$, which is appropriate within the distance of 5 Å required between the two catalytic residues for glucanase activity [50]. Similar positions of docked $\beta-(1,3)$-D-glycans have been reported on the surface of the modeled barley PR-5 protein HvPR5c [10], where the glucan chain lied in the middle of the catalytic pair. The distances between putative catalytic residues and the close $\beta$-(1,3)-glucan bond were $2.98 \AA$, $5.24 \AA$, $7.87 \AA$, and 7.92 Å for E84, D107, D112, and D207, respectively.

In the second case (Fig. 6B), the docking simulations demonstrated that Ole e 13 cleft is not big enough to accommodate the whole curdlan molecule, due to their large size and rigidity of the triple-helical structure and interactions, thus the rest of the glycan chain is positioned outside the cleft. This feature was also found for other TLPs [10]. The model predicts that the residues R44, G59, Q60, D65, G67, T76, Y77, R79, E84, D107, D112, T149, C160, P186, D199, D206, and D207are present within a distance about $3.5 \AA$ from the 

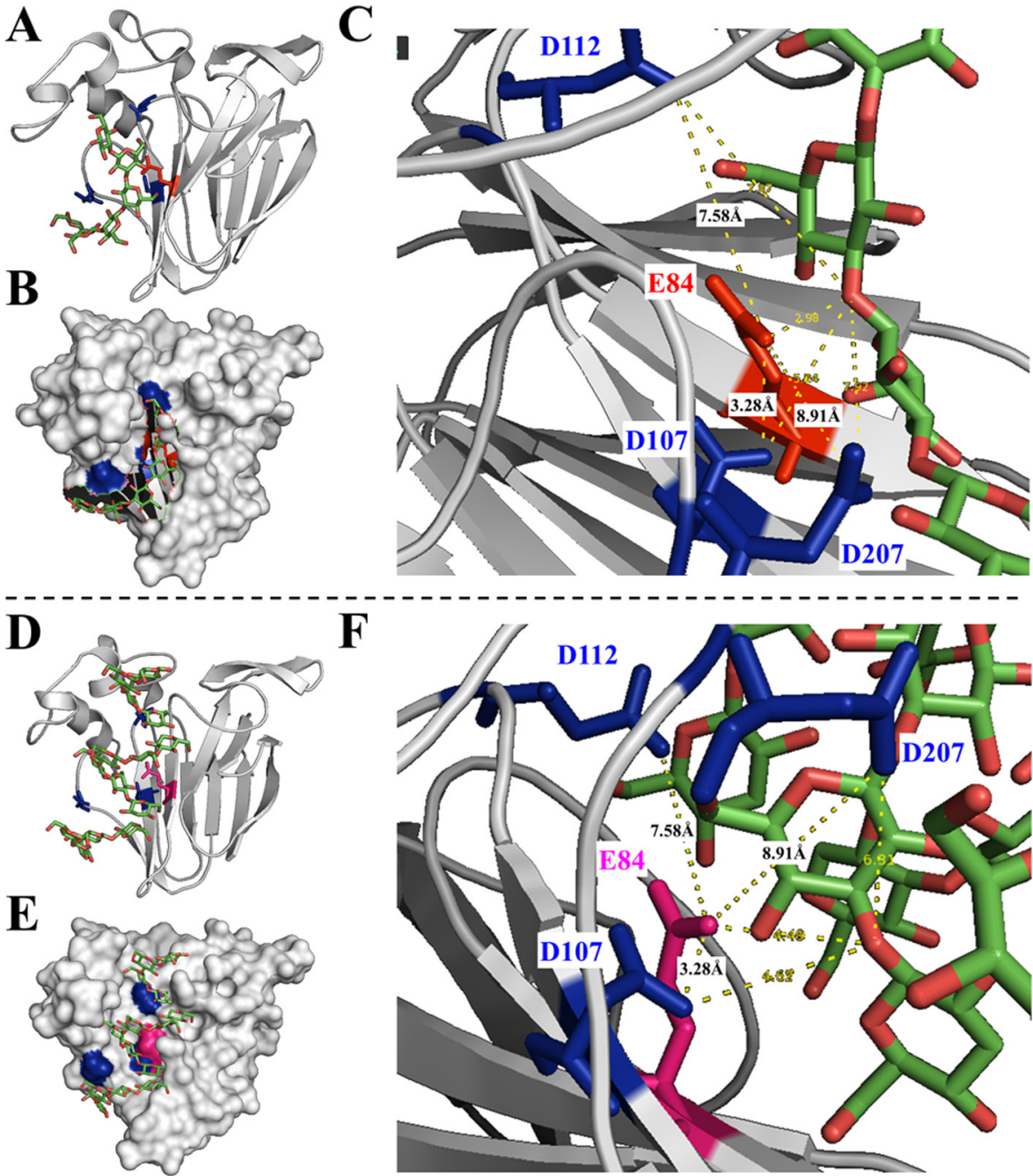

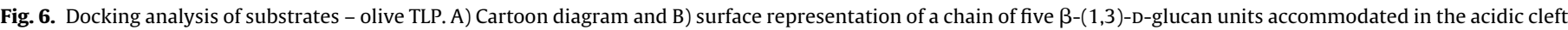

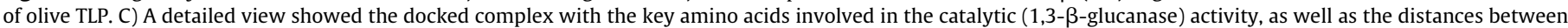

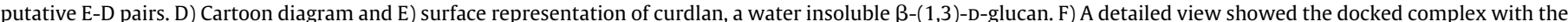

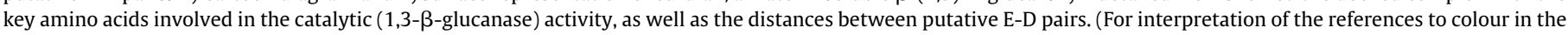
figure legend, the reader is referred to the web version of this article.)

docked glucan molecule. Among these residues, R44, G59, D65, W76, Y77, E84, D107, Thr149, C160, D206, and D207 are involved in hydrogen bonding with the bound polysaccharide. As happened in the previous docked glucan, the single curdlan $\beta-(1,3)$-D-glucan chain fits in the acidic cleft or passes through the central cleft region, and close (not in the middle of the pair) to E84 and D107. In this case the distance between $0 \varepsilon$ of E84 with $0 \delta$ of D107 is $3.28 \AA$, functioning as proton donor and nucleophile pair that acts as the plausible catalytic center required for endo-glucanase activity. The distances between catalytic residues and the close $\beta-(1,3)$-glucan bond were $4.49 \AA$, $4.62 \AA$. $6.31 \AA$, and $6.58 \AA$ to E84, D107, D112, and D207, respectively.

\subsection{Allergenicity assessment for Ole e 13}

Additionally to the antifungal and endo- $\beta$-1,3-glucanase activity, plant TLPs are getting an increasing interest because of their allergenic character [51]. Food allergy is a global concern, where particular fruit TLPs can cause class 1 food allergy, particularly due to a high resistance to denaturation and to proteolytic digestion [45], in great part due to the large number of disulfide bridges in their structures.
According to the FAO/WHO guidelines about allergenicity assessment, a protein is considered a potential cross-reactive allergen if the identity with the match of the full length allergen protein is $>50 \%$ or $>35 \%$ with an 80 amino acids window (80-mer) Searching with an 8 amino acids window, a $100 \%$ match is also considered a potential cross-reactive allergen.

Searching Ole e 13 , the sequence was about 78 and $83 \%$ of identity for full-length and 80-mer respectively. Thus, Ole e 13 can be considered as an allergen and a cross-reactive allergenic protein. A comparative search was also performed for several other allergens analyzed in the current study. Like this, we detected that Act d 2, Jun a 3, Cry j 3, were in the range of 53-69\% values for full-length, and $62-75 \%$ values for the 80 -mer method.

\subsection{IgE-binding epitopes prediction of olive Ole e 13 sequence}

Different members of the TLP family have been shown to have an important role in cross-allergenicity [52]. They do not only elicit food allergy (i.e. Pru av 2 from cherries [53], Cap a 1 from bell pepper [28], Mal d 2 from apple [45], and Act d 2 from kiwi [26,54]), but also class 2-allergy, as pollen allergens like Jun a 3 (mountain cedar) [21], 
Table 1

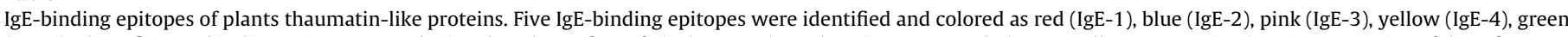

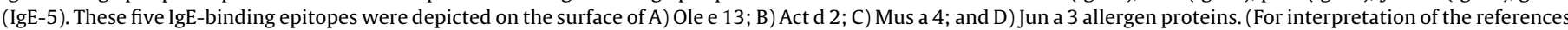
to colour in this table legend, the reader is referred to the web version of this article.)

\begin{tabular}{|c|c|c|c|c|c|}
\hline Specie/allergen & Epitope 1(46-57) & Epitope 2 (60-73) & Epitope 3 (137-148) & Epitope 4 (149-164) & Epitope 5 (176-191) \\
\hline Olea europaea E3SU11 & APGTTQARIWGRT & NFDANGRGQCETG & - & - & - \\
\hline Thaumatococcus daniellii_P02884 & - & YFDDSGRGICRTG & - & PGGGCNDAC'VFQ & - \\
\hline Solanum lycopersicum_P12670 & PRGTKMARIWGRT & NFNAAGRGTCQTG & - & - & - \\
\hline Nicotiana tabacum_P14170 & PRGTKMARVWGRT & NFNAAGRGTCQTG & - & - & - \\
\hline Nicotiana tabacum_Q01591 & PRGTKMARIWGRT & NFDGDGRGSCQTG & - & - & - \\
\hline Capsicum annuum_Q9ARG0 & PPGTAMARIWGRT & NFDGSGRGSCQTG & - & - & - \\
\hline Nicotiana tabacum_P25871 & PPGTKMARIWGRT & NFDGAGRGWCQTG & - & - & - \\
\hline Actinidia deliciosa_L7TUJ8 & GAGTKGARVWPRT & NFDGAGRGKCQTG & ADINGQCPNELR & APGGCNNPCTVFKT & - \\
\hline Musa accuminta_I3RTU5 & - & SFDGSGSGSCQTG & ADINGRCPAELR & TPGGCNNPCTYFIT & - \\
\hline Zea mays_P33679 & PAGTTAARIWART & KFDASGRGSCRTG & - & QDGVCNINACPVFIKK & - \\
\hline Oryza sativa_P31110 & PAGTSSGRVWGRT & SFDGSGRGSCATG & - & - & - \\
\hline Juniperus ashei_P81295 & AAGTASARFWGRT & TFDASGKGSCQTG & ADINAVCPSELK & VDGGCNSACNVFT' & NAYVDNCPATNYSK \\
\hline Prunus avium_P50694 & - & - & ANVNAVCPSELQ & - & - \\
\hline Malus domestica_Q3BCT8 & APSPWSGRFWGRT & - & - & - & - \\
\hline Arabiodpsis thaliana_P50699 & - & TFDRSGRGHCATG & - & - & - \\
\hline
\end{tabular}

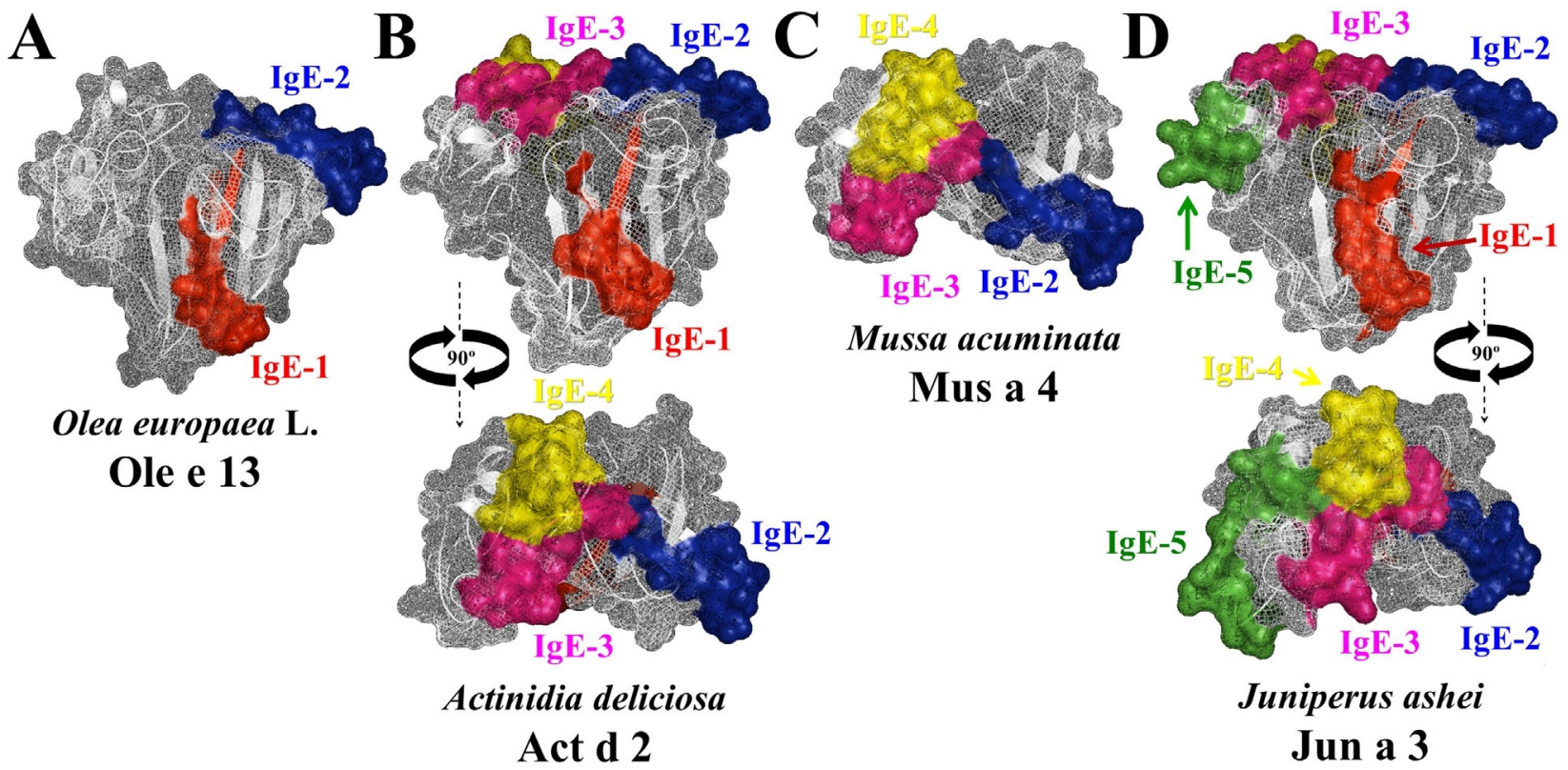

Cry j 1 (Japanese cedar) [23,55], Cup a 3 (Arizona cypress) [22,56], and Jun $v 3$ (Eastern red cedar) [57].

The presence of structurally well-conserved IgE binding epitopes with high percentage of conserved residues in their sequences might trigger cross-reactivity between food and pollen allergenic TLP. Such cross-reactivity between pollen and food allergens often occurs and is well documented [53,58]. A recognized major fruit allergen Mus a 4 allergen shares common conserved IgE-binding epitopes not only with other fruit TLPs as olive Ole e 13 (epitopes 2 and 4), cherry Pru av 2 allergen (epitope 3), kiwi Act d 2 (epitopes 2, 3 and 4), and thaumatin from T. daniellii (epitopes 2 and 4 ), but also with pollen mountain cedar Jun a 3 allergen (epitopes 2 , 3 and 4 ) (Table 1 ), but only recombinant forms of TLPs from apple and cherry have tested positive for IgE-reactivity with allergic sera [27,53].

IgE-binding epitopes were predicted for Ole e 13 and each one of the plant TLP sequences listed as allergens in Table 1 by using AlgPred prediction server. Location of these IgE-binding epitopes in the surface of Ole e 13 was depicted in Fig. 7. This analysis using a wide representation of pollen and fruit thaumatin-like pro- 


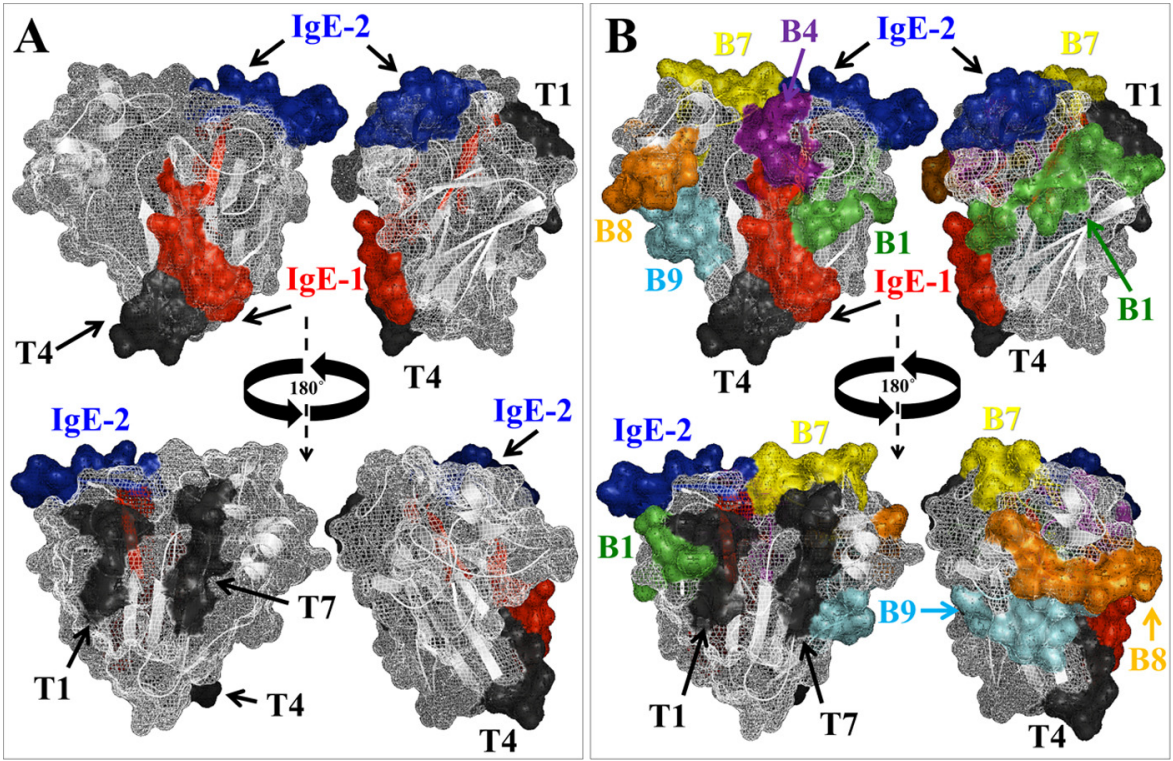

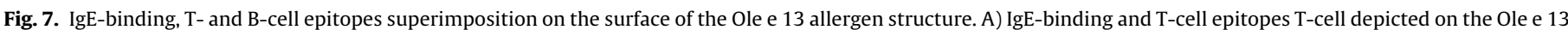

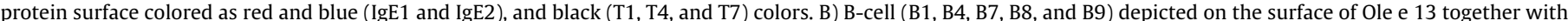
IgE-binding and T-cell epitopes. (For interpretation of the references to colour in this figure legend, the reader is referred to the web version of this article.)

teins allowed reaching numerous conclusions: i) the number of predicted IgE epitopes ranged from 1 to 5 concerning the species studied, ii) IgE-binding epitopes 1 ( 11 out of 15 sequences $=74.34 \%$ ) and 2 ( 13 out of 15 sequences $=86.67 \%$ ) are present in most of the sequences, iii) only a pollen TLP sequence displayed the five IgE epitopes (Juniperus ashei, Jun a 3 allergen protein); iv) only a food TLP sequence displayed the first four IgE-binding epitopes (Act $\mathrm{d}$ 2 allergen), and two sequences (Arabiodpsis thaliana, and Prunus avium) displayed only one IgE-binding epitope, number 2 and 3 , respectively; v) most of the IgE epitopes are located in the domain II (epitopes 3, 4, and 5), whereas only one (epitope 1 ) was located in domain I and another one (epitope 2 ) was located in domain III (Fig. 7, Table 1).

Two main IgE binding epitopes were predicted for Olea europaea L. Ole e 13 , corresponding to the epitope 1 ( ${ }^{46}$ APGTTQARIWGRT $\left.{ }^{57}\right)$, and epitope $2\left({ }^{60}\right.$ NFDANGRGQCETG $\left.{ }^{73}\right)$. These epitopes are 13 amino acids long, and are located in the domains I and III, respectively of the Ole e 13 structure (Fig. 7A). These two epitopes are in separate regions of the protein structure (Fig. 7B) when compared to $\mathrm{T}$ - and B-cell epitopes. IgE-binding epitope 1 of Ole e 13 share higher similarity (10 out of 13 residues $=76.92 \%$ ) to C. annuum (Cap a 1 ) and $N$. tabacum (PR5d), and a $69.23 \%$ of identity (9 out of 13 residues) with most of the thaumatin-like proteins. As regard to the epitope 2 , it overlaps with the thaumatin family signature as shown in Fig. 1. IgE-binding epitopes 1 and 2 are partially overlapping with B-cell epitopes 2 and 3. Prediction of these B-cell epitopes by BepiPred analysis showed that the residues having high scores are APGST and QGKC, respectively. These stretches found in IgE epitope indicate their high accessibility for antibody recognition.

Structure-based epitopes prediction has proved to be important method to identified key antigenic regions in allergen proteins [59]. It is important to build the structures of these allergens that have not been crystalized, as it is the case of Ole e 13. In this regard, apple Mal d 2, an orthologous of the major PR-10 allergen from birch (Betula verrucosa) pollen, has been identified as a TLP closely related structurally to TLP of Pru av 2 [25,53]. Cross-reactivity between Ole e 13 and other pollen and food allergens is likely to occur as the result of the large sequence identity. Thus, Ole e 13 and Jun a 3 share $70 \%$, and $62 \%$ of sequence identity in their epitopes 1 and 2 , respectively. Ole e 13 and Mal d 2 share $54 \%$ of sequence identity in epitope 1 . Moreover, Jun a 3 and Mal d 2 display the ability to bind Ig-E from the respective allergic individuals [25,27]. Ole e 13, Pru av 2 and Mal $\mathrm{d} 2$ share a similar a three-dimensional fold domain II, and these epitopes of Jun a 3, Act d 2 or thaumatin (Fig. 3A, Table 1). Moreover, the amino acid sequence of this $\alpha$-helical region share high identity to Jun a 3 and Mus a 4 (Fig. 1) and is predicted as a main IgE-binding epitope area. Mus a 4 is another example that retain structurally conserved IgE-binding epitopes (Fig. 1, Table 1) and hence might have a high chance of eliciting cross-reactive allergenic responses in individuals primary sensitized to pollen containing similar TLPs [30,51]. All these predicted regions include a 4-helix of the domain II, which is a 2-D structural element well conserved in Jun a 3 and others TLPs like Ole e 13, in addition to four conserved disulphide bridges C121-C173, C129-C139, C143-C152, and C153C160 (Ole e 13 positions in the 3D model) that highly stabilize the conformation of these IgE-binding epitopes.

\subsection{T-cell epitopes prediction of olive Ole e 13 and other plant TLPS}

T-cell epitopes play a central role in the cell mechanisms underlying the pathophysiology of different types of pollen and food allergies [60-64]. Teen different amino acid sequences namely T1 to $\mathrm{T} 10$ were identified among the thaumatin-like proteins analyzed (see Supplementry Table S1 in the online version at DOI: 10.1016/j. jmgm.2016.03.003a). However, in Ole e 13 only three of them (T1: 6-14, T4: 95-103, T7: 133-141) were predicted to be present (Fig. 7 , Table S1). These three T-cell epitopes were located exclusively in the domain I, T4 comprising part of the acidic cleft, while T1 and T7 in the opposite situation. Overall, and as an important observation, the location of T-cell epitopes in the allergen proteins of the other species mainly coincides in the domain I, overlapping small parts of the epitopes T1, T7, and T10 in the domains II and III (Table S1). The identified teen T-cell epitopes comprised 99 residues. As a counterpart, Ole e 13 integrated 27 residues, with a high frequency of occurrence for $\mathrm{N}$ ( 5 out of 27 residues $=18.52 \%$ ) and three residues $\mathrm{Q}$ and L. (11.12\%). $48.15 \%$ of the total residues were non polar whereas $33.33 \%$ were polar charged residues. Polar non-charged residues (18.52\%) were least preferred in the T-cell epitopes. All four T-cell epitopes are primarily composed by parts of $\alpha$-helices and loops. 
Surface distribution of T-cell epitopes were superimposed in the structure of Ole e 13 (Fig. 7) and other TLPs and depicted in Fig. 7 and Table S1.

Comparison of T-cell epitopes between the species analyzed yielded the following conclusions: Ole e 13 exhibited two specie-specific epitopes, T4 and T7. Similarly, specie-specific T-cell epitopes were detected for Pru av 2, Ara t TLP and Mus a 4 (T2, T8, and T10, respectively). Finally, the most commonly shared epitopes were $\mathrm{T} 1, \mathrm{~T} 5$, and $\mathrm{T} 10$.

\subsection{B-cell epitopes prediction of olive Ole e 12 sequence}

B-cell epitopes have been largely involved in driving the interaction of food allergens to specific IgE on antigen presenting cells such as basophils and mast cells. In addition, some B-cell epitopes are recognized more often than others, thus these highly recognized epitopes might play a major role in the sensitization process [61-65]. A total number of ten B-cell epitopes were identified among the fifteen thaumatin-like allergenic proteins studied (see Supplementry Table S2 in the online version at DOI: 10.1016/j. jmgm.2016.03.003a). C. annuum Cap a 1 allergen showed the larger number of B-cell epitopes (nine), followed by various other TLPs with eight B-cell epitopes. O. sativa_P31110 was the TLP with the lower number of B-cell epitopes (four). B-cell epitopes number 5 and 10 have the least representation among TLPs, only displayed in 3 (Jun a 3, Pru av 2 and Mal d 2) and 4 (Mus a 4, Cap a 1 and both $N$. tabacum sequences) allergenic proteins, respectively. Overall distribution of B-cell epitopes is not overlapping with IgE or T-cell epitopes, with the exception of $\beta 2, \beta 3, \beta 6$, and $\beta 10$ (Fig. 1 , Tables $1, \mathrm{~S} 1$ and $\mathrm{S} 2$ ).

A total number of seven B-cell epitopes, named sequentially as b1 to b4 and b7 to b9, spanning the entire length of Ole e 13 were predicted (Table S2). These seven regions are $\beta 1: 26-37, \beta 2: 43-47$, $\beta 3: 58-68, \beta 4: 83-87, \beta 7: 144-158, \beta 8: 173-184$, and $\beta 9: 197-206$ (Fig. 7B, Table S2) were identified as promiscuous binders by comparison of prediction data. The predicted epitopes comprised of 90 residues with a high frequency of occurrence for $\mathrm{A}(7.78 \%), \mathrm{F}$ (13.34\%), G (15.56\%). Hydrophobic content was analyzed to identify regions with a higher probability of interaction with immunoglobulin. Around $57.65 \%$ of the 90 residues were non polar, $26.67 \%$ were charged residues and $24.35 \%$ were recorded as polar non-charged residues for the predicted epitopes.

It can be noticed from the epitope predictions that two of the predicted seven B-cell epitopes overlapped with the Ole e 13 IgE-binding epitopes. Same was observed for all other TLPs analyzed, with the exception of Pru av 2 allergen which, on the contrary, exhibited a specific T-cell epitope 2 . This scenario lead us to think that overlapping IgE epitopes 1 and 2 might be strongly involved in primary sensitization to food and pollen allergen, and later cross-allergenicity phenomena. This is the case of Cap a 1 from bell pepper that shows an IgE-mediated contact allergy in patients with the mugwort-birch-celery-spice syndrome [58,66].

\section{Conclusions}

Structure functional studies based in computational approaches have widely demonstrated to be a powerful tool and a fundamental methodology for successful overcoming the lack of protein crystallographic structures for modeling and assessing specific structural features of proteins, discovering new molecular enzymatic mechanisms and their regulation. Accordingly, structural studies of thaumatin-like proteins provide critical insights into the diverse functions and regulation of the PR5 complex family in pathogens defense and response to different biotic and abiotic stresses.
Furthermore, based on in silico structure modeling, we were able to compare Ole e 13 to other close allergenic TLPs from food (kiwi, banana, apple) and pollen (mountain cedar, cypress) known to cause food allergy upon ingestion, and cross-allergenicity between food and pollen allergenic TLPs. Comparison was made throughout identification of species-specific epitopes, i.e. Ole e 13 T-cell epitopes 4 and 7, and commonly shared epitopes, i.e. Ole e 13 IgE-binding epitopes 1 and 2. Cross-reactivity is an important problem for the diagnosis and treatment of allergy. Thus, the identification of the patterns of association between different allergen sources from pollen and foods is a priority because of its importance for understanding how allergy is triggered.

Outcomes of the current work include: (1) a comprehensive understanding of the structure of Ole e 13 protein, and structural similarities and differences of Ole e 13 to others TLPs, (2) an overview of the molecular environment of the acidic cleft of the Ole e 13 and other TLPs for the different substrate recognitions and interactions, (3) a proposed catalytic mechanism for Ole e 13, which is highly dependent of the conservation of a catalytic pair E-D, crucial for a nucleophilic attack (base) of the glucan anomeric carbon, and as proton donor (acid), (4) the structural and molecular basis for the identification of these epitopes responsible of cross allergenicity between food and pollen allergenic TLPs. These epitopes may significantly contribute to designing rational strategies for diagnosis and immunotherapy to food allergy.

\section{Competing interests}

The authors have declared that no competing interests exist.

\section{Acknowledgements}

This work was supported by European Research Program MARIE CURIE (FP7-PEOPLE-2011-IOF), grant ref. number PIOF-GA-2011-301550 to JCJ-L and JDA; ERDF-cofunded projects RTC-2015-4181-2 (MINECO), 201540E065 (CSIC) and P2010-AGR-6274 and P2011-CVI-7487 (Junta de Andalucía).

JCJ-L thanks Spanish Ministry of Economy and Competitiveness for the grant ref. number RYC-2014-16536 (Ramon y Cajal Research Program).

\section{References}

[1] R. Velazhahan, S.K. Datta, S. Muthukrishnan, The PR-5 family: thaumatin-like proteins in plants, in: S.K. Datta, S. Muthukrishnan (Eds.),

Pathogenesis-related Proteins in Plants, CRC Press, Boca Raton, 1999, pp. $107-129$.

[2] J.J. Liu, A. Zamani, A.K.M. Ekramoddoullah, Expression profiling of a complex thaumatin-like protein family in western white pine, Planta 231 (2010) $637-651$.

[3] D.B. Tattersall, R. Van Heeswijck, P.B. Høj, Identification and characterization of a fruit-specific: thaumatin-like protein that accumulates at very high levels in conjunction with the onset of sugar accumulation and berry softening in grapes, Plant Physiol. 114 (1997) 759-769.

[4] A.B. Christensen, B.H. Cho, M. Naesby, P.L. Gregersen, J. Brandt, K. Madrid-Ordenana, D.B. Collinge, H. Thordal-Christensen, The molecular characterization of two barley proteins establishes the novel PR-17 family of pathogenesis-related proteins, Mol. Plant Pathol. 3 (2002) 135-144.

[5] L.C. Van Loon, M. Rep, C.M. Pieterse, Significance of inducible defense-related proteins in infected plants, Annu. Rev. Phytopathol. 44 (2006) 135-162.

[6] W.K. Roberts, C.P. Selitrennikoff, Zeamatin, an antifungal protein from maize with membrane-permeabilizing activity, J. Gen. Microbiol. 136 (1990) 1771-1778.

[7] R.W. Skadsen, P. Sathish, H.F. Kaeppler, Expression of thaumatin-like permatin PR-5 genes switches from the ovary wall to the aleurone in developing barley and oat seeds, Plant Sci. 156 (2000) 11-22.

[8] A.J. Vigers, W.K. Roberts, C.P. Selitrennikoff, A new family of plant antifungal proteins, Mol. Plant Microbe Interact. 4 (1991) 315-323.

[9] E. Vandermarliere, W. Lammens, J. Schoepe, S. Rombouts, E. Fierens, K. Gebruers, G. Volckaert, A. Rabijns, J.A. Delcour, S.V. Strelkov, C.M. Courtin, Crystal structure of the noncompetitive xylanase inhibitor TLXI: member of the small thaumatin-like protein family, Proteins 78 (2010) 2391-2394. 
[10] R.I.W. Osmond, M. Hrmova, F. Fontaine, A. Imberty, G.B. Fincher, Binding interactions between barley thaumatin-like proteins and $(1,3)-\beta$-D-glucans, Eur. J. Biochem. 15 (2001) 4190-4199.

[11] J. Trudel, J. Grenier, C. Potvin, A. Asselin, Several thaumatin-like proteins bind to ß-1, 3-glucans, Plant Physiol. 118 (1998) 1431-1438.

[12] J. Grenier, C. Potvin, J. Trudel, A. Asselin, Some thaumatin-like proteins hydrolyse polymeric ß-1,3-glucans, Plant J. 19 (1999) 473-480.

[13] H. Sassa, H. Hirano, Style-specific and developmentally regulated accumulation of a glycosylated thaumatin/PR5-like protein in Japanese pear (Pyrus serotina Rehd.), Planta 205 (1998) 514-521.

[14] L.A. Kempema, X.P. Cui, F.M. Holzer, L.L. Walling, Arabidopsis transcriptome changes in response to phloem-feeding silver leaf whitefly nymphs. Similarities and distinctions in responses to aphids, Plant Physiol. 143 (2007) 849-865.

[15] M.A. Batalia, A.F. Monzingo, S. Ernst, W. Roberts, J.D. Robertus, The crystal structure of the antifungal protein zeamatin, a member of the thaumatin-like, PR-5 protein family, Nat. Struct. Biol. 3 (1996) 19-23.

[16] E. Fierens, S. Rombouts, K. Gebruers, H. Goesaert, K. Brijs, J. Beaugrand, G. Volckaert, S. Van Campenhout, P. Proost, C.M. Courtin, J.A. Delcour, TLXI, a novel type of xylanase inhibitor from wheat (Triticum aestivum) belonging to the thaumatin family, Biochem. J. 403 (2007) 583-591.

[17] L. Menu-Bouaouiche, C. Vriet, W.J. Peumans, A. Barre, E.J.M. Van Damme, P. Rouge, A molecular basis for the endo-beta 1,3-glucanase activity of the thaumatin-like proteins from edible fruits, Biochimie 85 (2003) 123-131.

[18] R. Schimoler-O'Rourke, M. Richardson, C.P. Selitrennikoff1, Zeamatin inhibits trypsin and $\alpha$-Amylase activities, Appl. Environ. Microbiol. 67 (2001) 2365-2366

[19] M. Schraudner, D. Ernst, C. Langebartels, H. Sandermann, Biochemical plant responses to ozone: III. Activation of the defense-related proteins beta-1,3-glucanase and chitinase in tobacco leaves, Plant Physiol. 99 (1992) 1321-1328.

[20] H. Breiteneder, Thaumatin-like proteins-a new family of pollen and fruit allergens, Allergy 59 (2004) 479-481.

[21] T. Midoro-Horiuti, R.M. Goldblum, A. Kurosky, T.G. Wood, E.G. Brooks, Variable expression of pathogenesis-related protein allergen in mountain cedar (Juniperus ashei) pollen, J. Immunol. 164 (2000) 2188-2192.

[22] I. Cortegano, E. Civantos, E. Aceituno, A. del Moral, E. Lopez, M. Lombardero, V. del Pozo, C. Lahoz, Cloning and expression of a major allergen from Cupressus arizonica pollen Cup a 3, a PR-5 protein expressed under polluted environment, Allergy 59 (2004) 485-490.

[23] T. Fujimura, N. Futamura, T. Midoro-Horiuti, A. Togawa, R.M. Goldblum, H. Yasueda, A. Saito, K. Shinohara, K. Masuda, K. Kurata, M. Sakaguchi, Isolation and characterization of native Cry $\mathrm{j} 3$ from Japanese cedar (Cryptomeria japonica) pollen, Allergy 62 (2007) 547-553.

[24] R. Pressey, Two isoforms of NP24: a thaumatin-like protein in tomato fruit, Phytochemistry 44 (1997) 1241-1245.

[25] C. Inschlag, K. Hoffmann-Sommergruber, G. O’Riordain, H. Ahorn, C. Ebner, O. Scheiner, H. Breiteneder, Biochemical characterization of Pru av 2, a 23-kD thaumatin-like protein representing a potential major allergen in cherry (Prunus avium), Int. Arch. Allergy Immunol. 116 (1998) 22-28.

[26] M. Gavrovic-Jankulovic, T. Cirkovic, O. Vuckovic, M. Atanaskovic-Markovic, A Petersen, G. Gojgic, L. Burazer, R.M. Jankov, Isolation and biochemical characterization of a thaumatin-like kiwi allergen, J. Allergy Clin. Immunol. 110 (2002) 805-810.

[27] M. Krebitz, B. Wagner, F. Ferreira, C. Peterbauer, N. Campillo, M. Witty, D Kolarich, H. Steinkellner, O. Scheiner, H. Breiteneder, Plant-based heterologous expression of Mal d 2 a thaumatin-like protein and allergen of apple (Malus domestica), and its characterization as an antifungal protein, J. Mol. Biol. 329 (2003) 721-730

[28] H.C. Fuchs, K. Hoffmann-Sommergruber, B. Wagner, M. Krebitz, O. Scheiner H. Breiteneder, Heterologous expression in Nicotiana benthamiana of Cap a 1 a thaumatin-like protein and major allergen from bell pepper (Capsicum annuum), J. Allergy Clin. Immunol. 109 (2002) 134-135.

[29] E.A. Pastorello, L. Farioli, V. Pravettoni, C. Ortolani, D. Fortunato, M.G. Giuffrida, L. Perono Garoffo, A.M. Calamari, O. Brenna, A. Conti, Identification of grape and wine allergens as an endochitinase 4, a lipid-transfer protein, and a thaumatin. J. Allergy Clin. Immunol. 111 (2003) 350-359.

[30] P. Leone, L. Menu-Bouaouiche, W.J. Peumans, F. Payan, A. Barre, A. Roussel, E.J Van Damme, P. Rouge, Resolution of the structure of the allergenic and antifungal banana fruit thaumatin-like protein at 1.7- $\AA$, Biochimie 88 (2006) 45-52.

[31] M. Hauser, A. Roulias, F. Ferreira, M. Egger, Panallergens and their impact on the allergic patient, Allergy Asthma Clin. Immunol. 6 (2010) 1.

[32] A. Grosdidier, V. Zoete, O. Michielin, SwissDock a protein-small molecule docking web service based on EADock DSS, Nucleic Acids Res. 39 (2011) 270-277.

[33] E. Mashiach, D. Schneidman-Duhovny, N. Andrusier, R. Nussinov, H.J. Wolfson, FireDock: a web server for fast interaction refinement in molecular docking, Nucleic Acids Res. 36 (2008) 229-232.

[34] D. Schneidman-Duhovny, Y. Inbar, R. Nussinov, H.J. Wolfson, PatchDock and SymmDock: servers for rigid and symmetric docking, Nucleic Acids Res. 33 (2005) 363-367

[35] S.K. Jami, T. Swathi Anuradha, L. Guruprasad, P.B. Kirti, Molecular: biochemical and structural characterization of osmotin- like protein from black nightshade (Solanum nigrum), J. Plant Physiol. 164 (2007) 238-252.
[36] H. Tachi, K. Fukuda-Yamada, T. Kojima, M. Shiraiwa, H. Takahara, Molecular characterization of a novel soybean gene encoding a neutral PR-5 protein induced by high-salt stress, Plant Physiol. Biochem. 47 (2009) 73-79.

[37] H. Koiwa, H. Kato, T. Nakatsu, J. Oda, Y. Yamada, F. Sato, Crystal structure of tobacco PR-5d protein at 1.8 Å resolution reveals a conserved acidic cleft structure in antifungal thaumatin-like proteins, J. Mol. Biol. 286 (1999) 1137-1145.

[38] K. Min, S.C. Ha, P.M. Hasegawa, R.A. Bressan, D.-J. Yun, K.K. Kim, Crystal structure of osmotin: a plant antifungal protein, Proteins Struct. Funct. Bioinform. 54 (2004) 170-173.

[39] R. Ghosh, C. Chakrabarti, Crystal structure analysis of NP24-I: a thaumatin-like protein, Planta 228 (2008) 883-890.

[40] R. Kaneko, N. Kitabatake, Structure-sweetness relationship in thaumatin: importance of lysine residues, Chem. Senses 26 (2001) 167-177.

[41] T. Masuda, K. Ohta, B. Mikami, N. Kitabatake, High-resolution structure of the recombinant sweet-tasting protein thaumatin I, Acta Crystallogr. Sect. F Struct. Biol. Cryst. Commun. 67 (2011) 652-658.

[42] J.M. Slootstra, P. De Geus, H. Haas, C.T. Verrips, R.H. Meloen, Possible active site of the sweet-tasting protein thaumatin, Chem. Senses 20 (1995) 536-543.

[43] S. Rombouts, E. Fierens, E. Vandermarliere, A. Voet, K. Gebruers, J. Beaugrand, C.M. Courtin, J.A. Delcour, M. de Maeyer, A. Rabijns, S. Van Campenhout, G. Volckaert, His22 of TLXI plays a critical role in the inhibition of glycoside hydrolase family 11 xylanases, J. Enzyme Inhib. Med. Chem. 24 (2009) 38-46.

[44] E. Fierens, K. Gebruers, A.R. Voet, M. De Maeyer, C.M. Courtin, J.A. Delcour, Biochemical and structural characterization of TLXI, the Triticum aestivum L. thaumatin-like xylanase inhibitor, J. Enzyme Inhib. Med. Chem. 24 (2009) 646-654.

[45] U. Smole, M. Bublin, C. Radauer, C. Ebner, H. Breiteneder, Mal d 2 the thaumatin-like allergen from apple, is highly resistant to gastrointestinal digestion and thermal processing, Int. Arch. Allergy Immunol. 147 (2008) 289-298.

[46] A. Brandazza, S. Angeli, M. Tegoni, C. Cambillau, P. Pelosi, Plant stress proteins of the thaumatin-like family discovered in animals, FEBS Lett. 57 (2004) $3-7$.

[47] S. Anzlovar, M. Dalla Serra, M. Dermastia, G. Menestrina, Membrane permeabilizing activity of pathogenesis-Related protein linusitin from flax seed, Mol. Plant Microbe Interact. 11 (1998) 610-617.

[48] T. Theis, U. Stahl, Antifungal proteins: targets: mechanisms and prospective applications, Cell Mol. Life Sci. 61 (2004) 437-455.

[49] E.J. Van Damme, D. Charels, L. Menu-Bouaouiche, P. Proost, A. Barre, P. Rouge, W.J. Peumans, Biochemical: molecular and structural analysis of multiple thaumatin-like proteins from the elderberry tree (Sambucus nigra L.), Planta 214 (2002) 853-862.

[50] E.L. Zechel, S.G. Withers, Dissection of nucleophilic and acid-base catalysis in glycosidases, Curr. Opin. Chem. Biol. 5 (2001) 643-649.

[51] T. Midoro-Horiuti, E.G. Brooks, R.M. Goldblum, Pathogenesis-related proteins of plants as allergens, Ann. Allergy Asthma Immunol. 87 (2001) 261-271.

[52] A. Palacín, L.A. Rivas, C. Gómez-Casado, J. Aguirre, L. Tordesillas, J. Bartra, C. Blanco, T. Carrillo, J. Cuesta-Herranz, J.A. Bonny, E. Flores, M.G. García-Alvarez-Eire, I. García-Nuñez, F.J. Fernández, P. Gamboa, R. Muñoz, R. Sanchez-Monge, M. Torres, S.V. Losada, M. Villalba, F. Vega, V. Parro, M. Blanca, G. Salcedo, A. Díaz-Perales, The involvement of thaumatin-like proteins in plant food cross-reactivity: a multicenter study using a specific protein microarray, PLoS One 7 (2012) e44088.

[53] H.C. Fuchs, B. Bohle, Y. Dall'Antonia, C. Radauer, K. Hoffmann-Sommergruber, A. Mari, O. Scheiner, W. Keller, H. Breiteneder, Natural and recombinant molecules of the cherry allergen Pru av 2 show diverse structural and B cell characteristics but similar T cell reactivity, Clin. Exp. Allergy 36 (2006) 359-368.

[54] A. Palacin, J. Rodriguez, C. Blanco, G. Lopez-Torrejon, R. Sanchez-Monge, J. Varela, M.A. Jimenez, J. Cumplido, T. Carrillo, J.F. Salcedo, Immunoglobulin e recognition patterns to purified kiwifruit (Actinidinia deliciosa) allergens in patients sensitized to kiwi with different clinical symptoms, Clin. Exp. Allergy 38 (2008) 1220-1228.

[55] N. Futamura, Y. Mukai, M. Sakaguchi, H. Yasueda, S. Inouye, T. Midoro-Horiuti, R.M. Goldblum, K. Shinohara, Isolation and characterization of cDNAs that encode homologs of a pathogenesis-related protein allergen from Cryptomeria japonica, Biosci. Biotech. Bioch. 66 (2002) 2495-2500.

[56] I. Cortegano, E. Civantos, E. Aceituno, A. del Moral, E. Lopez, M. Lombardero, V. del Pozo, C. Lahoz, Cloning and expression of a major allergen from Cupressus arizonica pollen Cup a 3, a PR-5 protein expressed under polluted environment, Allergy 59 (2004) 485-490.

[57] T. Midoro-Horiuti, R.M. Goldblum, E.G. Brooks, Identification of mutations in the genes for the pollen allergens of eastern red cedar (Juniperus virginiana), Clin. Exp. Allergy 31 (2001) 771-778.

[58] A. Leitner, E. Jensen-Jarolim, R. Grimm, B. Wüthrich, H. Ebner, O. Scheiner, D. Kraft, C. Ebner, Allergens in pepper and paprika: immunologic investigation of the celery-birch-mugwort-spice syndrome, Allergy 53 (1998) 36-41.

[59] F. Dall'Antoniaa, T. Pavkov-Kellerb, K. Zanggerd, W. Keller, Structure of allergens and structure based epitope predictions, Methods 66 (2014) $3-21$.

[60] B. Bohle, T-cell epitopes of food allergens, Clin. Rev. Allergy Immunol. 30 (2006) $97-108$ 
[61] O. Palomares, The role of regulatory T cells in IgE-mediated food allergy, J. Investig. Allergol. Clin. Immunol. 23 (2013) 371-382.

[62] J.C. Jimenez-Lopez, S.O. Kotchoni, M.C. Hernandez-Soriano, E.W. Gachomo, J.D. Alché, Structural functionality catalytic mechanism modeling and molecular allergenicity of phenylcoumaran benzylic ether reductase, an olive pollen (Ole e 12) allergen, J. Comput. Aided Mol. Des. 27 (2013) 873-895.

[63] J.C. Jimenez-Lopez, S.O. Kotchoni, M.I. Rodríguez-García, J.D. Alché, Structure and functional features of olive pollen pectin methylesterase using homology modeling and molecular docking methods, J. Mol. Model. 18 (2012) 4965-4984.

[64] J.C. Jimenez-Lopez, M.I. Rodríguez-García, J.D. Alché, Analysis of the effects of polymorphism on pollen profilin structural functionality and the generation of conformational, T- and B-cell epitopes, PLoS One 8 (2013) e76066.
[65] R. Valenta, H. Hochwallner, B. Linhart, S. Pahr, Food allergies: the basics, Gastroenterology 148 (2015) 1120-1131.

[66] M.D. Anliker, S. Borelli, B. Wüthrich, Occupational protein contact dermatitis from spices in a butcher: a new presentation of the mugwort-spice syndrome, Contact Dermatitis 46 (2002) 72-74. 